\title{
La Conflictividad Social en Clave Local. Un Ejercicio de Ponderación sobre los hechos de Rebelión en La Mar Del Plata actual (Argentina, 2011-2016)
}

SISMOS 1

Resumen: En el artículo propuesto se presenta un avance de la investigación en curso sobre la conflictividad social en las comunidades portuarias del sudeste bonaerense durante el período 2011-2016. Analizamos el caso de la ciudad puerto de Mar del Plata. En primer lugar, se da cuenta de los distintos clivajes emergentes en los hechos de rebelión, dividiéndolos en dos grandes grupos: los hechos impulsados por personificaciones de la relación capitaltrabajo y los hechos emprendidos por personificaciones que se producen por fuera de la relación capital-trabajo. En un segundo lugar, se ensaya una ponderación de los conflictos con el objetivo de arriesgar una caracterización e interpretación global de la dinámica conflictual en aquellos años. La base empírica para dicho ejercicio es el registro normalizado que de la conflictividad social se viene realizando en el marco del Seminario de Investigación Sobre el Movimiento de la Sociedad (SISMOS), cuya fuente de información es el diario local La Capital.

\footnotetext{
${ }^{1}$ Seminario de Investigación Sobre el Movimiento de la Sociedad, Centro de Estudios Históricos, Universidad Nacional de Mar del Plata/Consejo Nacional de Investigaciones Científicas y Técnicas (CONICET). Integran SISMOS: Agustín Nieto, Guillermina Laitano, Nicolás Rabino, Luis Nogueira, Luciana Nogueira, Lucía Andreozzi, Pablo Becher, Luis Fabiani, Gabriela Sánchez, Ivana Teijón, Eliana Marioli, Emmanuel Pironi, Camila Okada. Página de internet: http://observatoriodeconflictividad.org Correo electrónico: basesdedatosmdp@gmail.com
}

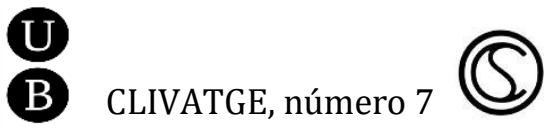


Palabras clave: conflictividad social, base de datos, ponderación, hechos de rebelión, Mar del Plata

Resum: En l'article proposat es presenta un avanç de la investigació en curs sobre la conflictivitat social a les comunitats portuàries del sud-est de Buenos Aires durant el període 2011-2016. Hi analitzem el cas de la ciutat port de Mar del Plata. En primer lloc, es dona compte dels diferents clivatges emergents en els fets de rebel-lió, que es divideixen en dos grans grups: els fets impulsats per personificacions de la relació capital-treball i els fets empresos per personificacions que es produeixen fora de la relació capital-treball. En segon lloc, s'hi assaja una ponderació dels conflictes amb l'objectiu d'arriscar una caracterització i interpretació global de la dinàmica conflictual d'aquells anys. La base empírica per a aquest exercici és el registre normalitzat que de la conflictivitat social es realitza en el marc del Seminari d'Investigació Sobre el Moviment de la Societat (SISMOS), la font d'informació del qual és el diari local La Capital.

Paraules clau: conflictivitat social, base de dades, ponderació, fets de rebel-lió, Mar del Plata

Abstract: In this article we present a preview of an ongoing investigation on social conflict situations in the port communities of southeast Buenos Aires in the period 2011-2016. We examine the case of the port city of Mar del Plata. Firstly, we present the several cleavages emerging in the conflicts and divide them in two large groups: capital-labour conflicts and conflicts which happen outside the capital-labour relations. In the second place, we attempt an analysis of the conflicts in order to propose a characterization and global interpretation of conflict dynamics in those years. The empirical base of this exercise is the normalized record of social conflict situations that we are preparing within the Research Seminar

B CLIVATGE, número 7

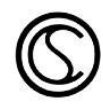


on the Movement of Society (SISMOS), whose source of information is the local newspaper La Capital.

Keywords: social conflict, database, pondering, rebellion events, Mar del Plata

\section{Introducción}

En el presente artículo presentamos un avance de la investigación en curso sobre la conflictividad social en las comunidades portuarias del sudeste bonaerense durante el período 2011-2016.² En esta ocasión analizamos particularmente el caso de Mar del Plata: es la ciudad cabecera del partido de Gral. Pueyrredón y está ubicada $404 \mathrm{~km}$ al sureste de la Ciudad Autónoma de Buenos Aires, capital federal de la República Argentina. En relación a su estructura productiva, sobresalen la industria turística y la actividad portuaria, pues la ciudad contiene al puerto pesquero más importante del país. Esta panorámica -muy general- se completa con una industria textil, la producción frutihortícola en la zona periurbana de la ciudad, y la producción agraria y ganadera en los campos que la circundan.

Mar del Plata fue y es una ciudad receptora de flujos migratorios internos y externos, rasgo que le imprime una morfología

\footnotetext{
2 Proyectos de investigación: PICT-2015-0121: "La conflictividad social en la Argentina contemporánea: procesos de lucha y organización en las ciudades-puerto del sudeste bonaerense, 2001-2008", Investigador responsable Dr. Agustín Nieto; “Procesos y ciclos de conflictividad social en la Argentina contemporánea. El caso de las ciudades-puerto del sudeste bonaerense: Mar del Plata, Quequén-Necochea e Ing. White-Bahía Blanca (20012008)" del Grupo de Estudios Sociales Marítimos, Facultad de Humanidades, UNMdP.
}

B CLIVATGE, número 7

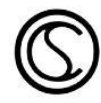


singular a su mercado de fuerza de trabajo. Este perfil, en un marco de crisis socio-económicas cíclicas como el de las últimas décadas, la sostiene como una ciudad con una tasa alta de desocupación. Las industrias textil y pesquera vienen arrastrando una crisis que en los últimos años se tornó crónica. Esta situación agrava la ya precaria condición asalariada de lxs trabajadorxs de estas ramas así como la del conjunto del proletariado de la ciudad y de la zona. De conjunto, la intensidad de la crisis no es constante y muestra vaivenes a lo largo de los años bajo estudio. Los momentos de crisis más aguda son seguidos por momentos de relativa reactivación que desembocan en una nueva agudización de la crisis. Esta situación no solo se manifiesta en los mercados de fuerza de trabajo sino también en las políticas públicas orientadas a la regulación de la fuerza de trabajo así como a aquellas orientadas a las poblaciones de los barrios con mayores índices de pobreza.

Toda esta situación tiene su contraparte conflictiva que la dinamiza. El proletariado del ámbito estatal es uno de los sectores con mayor tasa de activación conflictual del período. En particular, lxs trabajadorxs del poder judicial, lxs docentes, lxs trabajadores de dependencias gubernamentales, lxs médicxs de los hospitales son quienes tienen una mayor presencia en las calles con comunicados, manifestaciones, asambleas, cortes de calle, huelgas, en reclamo de salarios e infraestructura. En los últimos años, las demandas de tipo ofensiva dejaron lugar a las demandas de tipo defensiva: pago de salarios atrasados, reincorporación de lxs despedidxs, reapertura de paritarias, entre otras. Algo similar ocurrió con las demandas en el

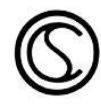


ámbito privado. En este ámbito, el protagonismo más destacado lo tuvieron lxs trabajadorxs de la industria pesquera, de la energía, del transporte, de la educación privada. Aunque tuvieron una presencia menor a la del proletariado del ámbito estatal, sus acciones tuvieron mayores repercusiones económicas. De conjunto, los conflictos protagonizados por el proletariado concitaron la participación y la mediación de lxs funcionarixs de los gobiernos local, provincial y nacional, así como de lxs funcionarixs de la iglesia católica.

Por fuera de la relación asalariada se destacaron los conflictos impulsados por organizaciones de vecinxs para el fomento barrial, grupo de vecinxs autoconvocadxs contra hechos delictivos en los barrios, organizaciones de mujeres, agrupaciones en defensa de los derechos humanos, comunidades educativas, agrupaciones estudiantiles, entre otras. Como veremos, este tipo de organizaciones emprendieron acciones de corte más radical en su formato por demandas tanto ofensivas como defensivas en torno a las condiciones de vida barrial y a las condiciones de estudio en las instituciones educativas. Las mediaciones político-institucionales estuvieron centradas en lxs funcionarixs municipales, en los agentes policiales y en las autoridades educativas.

En cuanto a los objetivos centrales del presente escrito, en primer lugar realizamos un abordaje descriptivo de la conflictividad marplatense en el periodo bajo estudio, dando cuenta de las formas de rebelión, pero partiendo de una primera división ordenadora: por un lado observamos los hechos de rebelión (en adelante HR) emprendidos por sujetos que son personificaciones de la relación

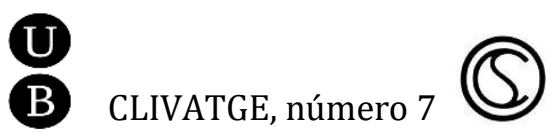


capital-trabajo (en adelante SPRK/T) y, por el otro, aquellos HR que son impulsados por sujetos que personifican otras relaciones sociales por fuera de relación K/T (en adelante SPORS). ${ }^{3}$ En segundo lugar, ensayamos una ponderación cualitativa de los HR con el objetivo de superar el plano meramente descriptivo y arriesgar una caracterización e interpretación global de la dinámica conflictual en aquellos años.

La base empírica para dicho ejercicio es el registro normalizado de la conflictividad social que se viene realizando en el marco del Seminario de Investigación Sobre el Movimiento de la Sociedad (en adelante SISMOS), cuya fuente de información es el diario local $L a$ Capital.

Dos cuestiones a tener en cuenta en función de lo antedicho. En primer lugar, es necesario aclarar por qué aplicamos como primera división ordenadora el tipo de sujeto que emprende los conflictos (es decir si son emprendidos por SPRK/T o por SPORS). Al igual que otros grupos de investigación, como el Programa de Investigación sobre el Movimiento de la Sociedad Argentina (en adelante PIMSA) y el Grupo de Estudios sobre Protesta Social y Acción Colectiva (GEPSAC), desde el SISMOS relevamos el conflicto social emprendido por el conjunto de personificaciones sociales, sin limitarnos a una sola de ellas. ${ }^{4}$

\footnotetext{
${ }^{3}$ Las siglas SPRK/T y SPORS en algunas ocasiones aparecen en el texto sin la S inicial, de "sujetos", en función de cada frase particular para respetar la coherencia de la frase en la escritura.

${ }^{4}$ El Ministerio de Trabajo, Empleo y Seguridad Social (MTEySS) y los observatorios como el Observatorio del Derecho Social de la CTA y los observatorios provinciales de Córdoba y
} 
Asimismo, en línea con la tradición teórica del PIMSA, entendemos que si bien la relación $\mathrm{K} / \mathrm{T}$ es una relación independiente de la voluntad de los individuos y estructurante de un orden social específico -esquemáticamente, están quienes viven de la venta de su fuerza de trabajo y quienes viven de la compra de la ajena-, lo cierto es que cuando observamos el conflicto, los sujetos que lo emprenden lo hacen desde alguna de las múltiples identidades que portan, donde la de asalariados/as o capitalistas es sólo una entre las posibles. Pues, los individuos son nudos de múltiples y diversas relaciones sociales.

Sin embargo, y en calidad de hipótesis de trabajo, la identidad de asalariados/as y capitalistas, por su peso relativo en el conjunto de los sujetos que emprenden hechos de rebelión, es una identidad preponderante. Esto es, en los datos se observa que si bien no es la única personificación social a partir de la cual los sujetos expresan los conflictos, sí es la principal en términos cuantitativos, tornándose pertinente de análisis específicos en términos cualitativos. Esto no es de extrañar en un orden social preponderantemente capitalista donde el conflicto entre capital y trabajo es co-constitutivo de dicho orden social. En este sentido, la historia de los conflictos cuyas personificaciones actuantes son los trabajadores/as es rica y compleja en su forma y contenido, para dimensionar su singularidad basta pensar que en su repertorio de instrumentos de lucha lista la huelga, una forma propia de la relación $\mathrm{K} / \mathrm{T}$, que no puede ser replicada por

Mendoza, entendiendo que es la conflictividad laboral la de mayor relevancia social, centran su análisis en los conflictos que se dan en el marco de las relaciones $\mathrm{K} / \mathrm{T}$.

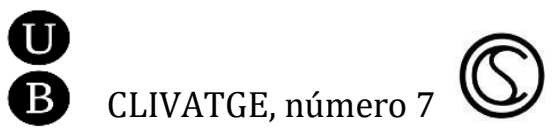


otras personificaciones sociales.

En segundo lugar, es necesario aclarar la relevancia que en términos teórico-metodológicos le otorgamos a la ponderación cualitativa de los datos. Las investigaciones cuantitativas sobre el conflicto social en Argentina se destacan por presentar análisis estadísticos descriptivos. En estos análisis predominan la exposición de frecuencias simples o bien el cruce dicotómico entre variables, pero son virtualmente inexistentes mediciones que permitan ponderar cualitativamente los datos cuantitativos sobre el conflicto social de modo de avanzar hacia el planteamiento de problemas en clave explicativa (Maañón y Nievas, 2003; Millán, 2007).

Anclarse en los estudios descriptivos no sólo no permite avanzar en la formulación de hipótesis explicativas, sino que también implica el riesgo de construir interpretaciones verosímiles, aunque erradas acerca del devenir de la lucha de clases. En otras palabras, el análisis unilateral de variables descriptivas puede traducirse fácilmente en una visión impresionista de los datos. Como señalan Maañón y Nievas (2003), el problema es que se asimila la contabilización de los datos a su medición, cuando en realidad son operaciones estadísticas diferentes en la medida en que las acciones registradas no son iguales. ${ }^{5}$

Estos riesgos existen en la medida en que aquello que se mide

\footnotetext{
${ }^{5}$ Un ejemplo ilustrativo de tal situación ocurre cuando se establece una equivalencia entre el número de acciones de rebelión y la intensidad de la rebelión, es decir, cuando se infiere que el aumento en términos absolutos de la cantidad de acciones registradas equivale por sí mismo a un aumento en la intensidad de la lucha de clases.
}

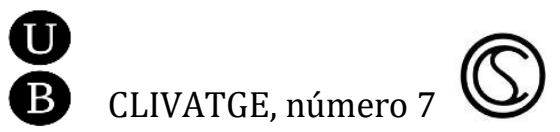


en este tipo de investigaciones sobre los procesos de rebelión presenta una amplia heterogeneidad de situaciones que el investigador debe homogeneizar para su análisis estadístico a partir de bases de datos. ${ }^{6}$ Por ello, es necesario desarrollar herramientas metodológicas que habiliten la restitución de aquellas heterogeneidades anuladas, en la medida en que son ellas las que nos permiten ponderar cualitativamente los datos cuantitativos. ${ }^{7}$

Finalmente, más allá de que en el tratamiento de los datos proponemos trabajar a partir de variables analíticas que ponderen la información, debemos mencionar aquí que nuestra propuesta metodológica incorpora elementos de ponderación desde la misma construcción de la base de datos. En efecto, si bien - como mencionamos - nuestra unidad de análisis son acciones, estas son encadenadas entre sí cuando refieren a un mismo conflicto, y se conforman en tanto hechos compuestos (en el apartado siguiente detallamos la operación). Según Franzosi (2017) la distribución de los eventos depende de sus vínculos estratégicos en cadena. Estos eventos en cadena "antes que eventos singulares, constituyen la real unidad de análisis". Pues, según el autor, la unidad significativa para

\footnotetext{
${ }^{6}$ Esta situación se visualiza ya en la definición de la unidad de análisis. En nuestro caso la unidad de análisis es la acción de rebelión definida en sentido amplio como acciones colectivas dirigidas contra alguna expresión del estado de cosas existente, de modo tal que incluye acciones tan heterogéneas como una declaración o conferencia de prensa hasta una toma de fábrica.

${ }^{7}$ Los primeros ejercicios de ponderación fueron presentados en Laitano, Rabino y Nieto (2015) y en Guixá, Laitano, Rabino y Nieto (2015).
}

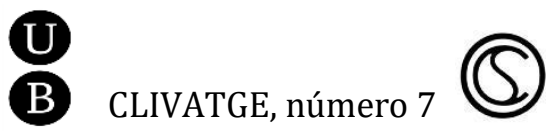


entender el conflicto laboral no es la huelga, concebida como interrupción de trabajo, sino la disputa que incluye no solo uno o más paros, sino también diferentes formas de lucha como manifestaciones, concentraciones, asambleas, reuniones de negociación, etc. En esta operación metodológica subyacen las sugerencias de Chakrabarty (2008), quien nos alerta acerca de los riesgos de reproducir en la reconstrucción de la historia de las clases subalternas el individualismo metodológico comúnmente aplicado a éstas por las prácticas de Estado o de las elites. Estas prácticas se caracterizan por psicologizar o individualizar los hechos de rebelión (puesto que sólo así pueden cumplir con el propósito de interrogar, responsabilizar y castigar), hechos que, por su carácter social, son necesariamente colectivos. En este sentido, pensamos que considerar cada acción de rebelión como una única unidad de medición del conflicto social conlleva el riesgo de inobservar su carácter procesual, cuyo desenvolvimiento involucra múltiples sujetos y formas que se determinan coyunturalmente, y no en un tiempo homogéneo y lineal.

Para ordenar la exposición del trabajo, en primer lugar, presentamos una breve referencia a la perspectiva teóricometodológica a partir de la cual abordamos el conflicto social como problemática de estudio desde el SISMOS. Seguidamente, retomando los trabajos del PIMSA, reseñamos la situación a nivel nacional del período bajo estudio, al tiempo que respondemos al interrogante acerca de por qué es necesaria la elaboración de una base de datos sobre el conflicto de carácter local, existiendo ya una base de carácter nacional que incluye a la ciudad de Mar del Plata. Finalmente, en los

B CLIVATGE, número 7

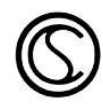


dos últimos apartados desarrollamos los objetivos propuestos en esta introducción. Primero, presentaremos los datos a partir de frecuencias simples y, luego, revisaremos los alcances explicativos de dichas frecuencias a partir de los ejercicios de ponderación.

\section{Contar y medir la conflictividad: notas teórico- metodológicas}

Desde el SISMOS elaboramos una base de datos cuyo objetivo general es registrar en forma estandarizada el universo de conflictos sociales de la ciudad de Mar del Plata visibilizados en la prensa. Para esa labor tomamos los ejemplares impresos del periódico de mayor circulación de la ciudad, el diario La Capital. La base de datos cuenta con una unidad de registro y de observación, que es cada noticia impresa individualizada para el relevamiento donde se haga referencia a una o más acciones conflictivas con independencia de la forma y lugar de su publicación. Vale aclarar que solo se relevan las notas que refieren a eventos iniciados en la ciudad, ya sea que aparezcan en las secciones "Policiales" o "La ciudad y la zona".8

\footnotetext{
${ }^{8}$ Sabemos los problemas y limitaciones que conlleva utilizar como fuente un periódico. Una de las limitaciones es que no lograremos registrar la "totalidad" de acciones conflictivas ocurridas en la ciudad. También sabemos que los criterios periodísticos y editoriales no son constantes ni homogéneos a lo largo del tiempo y que muchas veces responden a los tiempos políticos-institucionales. También estamos alertados de que la elección de cuántas y qué acciones se reseñan siempre depende de la política editorial y los intereses económicos de las empresas periodísticas. Buscamos superar esas limitaciones tomando solamente los datos duros que nos permiten establecer los valores de cada variable de la base de datos. Además, se trata de una fuente que registra cotidianamente "hechos" de una forma relativamente estandarizada, que tiene un control aunque sea mínimo de la
}

B CLIVATGE, número 7

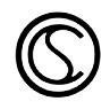


El esquema teórico-conceptual a partir del cual observamos y analizamos los procesos de organización y lucha en la ciudad de Mar del Plata se asienta principalmente en la teoría de los ciclos de rebelión en las sociedades contemporáneas, la cual precisa que el antagonismo social principal gira en torno a la relación K/T, entendida como una relación social de dominación y explotación (Wright, 2010). A su vez, nos enfocamos en la problemática de las situaciones y correlaciones de fuerzas sociales (Gramsci, 2010) en sus distintas dimensiones, correlaciones que dan lugar a heterogéneas configuraciones conflictuales y formas de resistencia (Edwards y Scullion, 1987).

Nuestra unidad de análisis es cada acción de rebelión descrita en cada una de las notas periodísticas, teniendo en cuenta todas las diferentes acciones de rebelión que pueda contener una misma nota; se define como todas aquellas acciones colectivas o individuales, llevadas a cabo por "personificaciones" de relaciones sociales "clasificables" como económicas, sociales o políticas, dirigida contra alguna expresión del estado de cosas existente. Por otra parte, aquellas acciones que están relacionadas espacial y temporalmente entre sí, ya sea por los actores que las llevan a cabo y los objetivos que

comunidad a la cual se dirige (no puede mentir sobre todo, todo el tiempo), además de contar con la ventaja de su conservación en hemerotecas de la ciudad así como en el archivo de la empresa periodística. Ver Ghigliani (2009); Sorribas (2010). Según Franzosi (2017) al utilizar la prensa como una fuente de datos, el riesgo que se corre no es el de recolectar información defectuosa sino insuficiente, razón por la cual el problema puede ser menos grave de lo que las investigaciones sobre medios de difusión nos han llegado a hacer creer.

B CLIVATGE, número 7

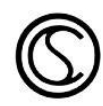


persiguen, como por las reacciones que generan en otros sujetos y los objetivos que puedan desplegar en relación al objetivo inicial, son agrupadas como "hechos compuestos de rebelión" ("eventos en cadena" en palabras de Franzosi), diferenciándolos de los "hechos simples" (que dan cuenta de una sola acción aislada).

A continuación describimos algunas de las variables utilizadas en nuestro registro:

- Forma de rebelión: es la variable principal de nuestra base de datos, describe el tipo de acción (su forma) en su máximo nivel de desagregación posible.

Variables temporales: en estas variables damos cuenta de la fecha de inicio y de finalización de las acciones de rebelión.

- Sujeto: esta variable tiene como objetivo registrar el valor de quién/es llevan adelante (impulsan) la acción de rebelión, definidos según el ámbito de relaciones sociales desde el cual se activan y movilizan. En cada acción registrada el sujeto que la emprende lo hace en tanto personificación de determinadas relaciones sociales más allá de que como individuo anude más relaciones sociales que las que se activan en la acción registrada.

- Tipo de Sujeto: en esta variable establecemos si el sujeto de la acción es o no personificación de relaciones $\mathrm{K} / \mathrm{T}$.

Otras variables son: tipo de organización; meta inmediata de la acción emprendida; destinatarixs directxs de la acción; variables espaciales, etc.

A su vez, nuestra base de datos cuenta con un Manual de Ponderación mediante el cual realizamos ejercicios de medición a 
partir de la recategorización de los valores extraídos de las variables descriptivas recién mencionadas. A continuación listamos algunas de las variables utilizadas para los ejercicios:

- Posición en la relación social general de dominación: esta variable referencia a los dos grandes campos de fuerza que tienden a ordenar al conjunto de los eventos conflictivos, a saber, campo del régimen y campo del pueblo (Balvé y Balvé, 1989).

- Caudal de conflictividad por período: esta variable permite medir la cantidad de conflictos que efectivamente se desarrollan en cada momento. También se puede medir el sentido de la tasa de actividad, es decir, si es creciente o decreciente. Así, permite establecer para el período analizado si la tendencia es a la reproducción, al consenso o al conflicto.

- Nivel de implicancia corporal en la acción: esta variable analítica busca establecer grados de implicación corporal de los sujetos (individuales o colectivos) en los procesos de lucha, dando por hecho que siempre existe un grado de implicancia (efectiva o potencial). Si bien toda tipología establece fronteras arbitrarias, lo que buscamos aquí es demarcar la implicancia corporal a partir de establecer grados de compromiso del cuerpo, riegos potenciales que corren los cuerpos en acción como consecuencia de dicha acción. Estos riesgos van desde la desaprobación de sus iguales hasta el riesgo de muerte. Si bien en cada acción cada uno de los tipos señalados (valores) puede estar presente, se trata de observar cuál es aquel nivel de implicancia del cuerpo que es predominante en cada acción. Es una variable ordinal. Es un indicador (aunque por sí sólo no basta) de la

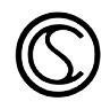


intensidad de la acción, lo que poco nos dice del poder efectivo de la acción en relación a los resultados buscados. La variable se elabora recategorizando los valores de la variable descriptiva "Forma de rebelión" y los nuevos valores son: baja (refiere a hechos discursivos, donde lo que predomina es el uso de la palabra, escrita o verbal, como en una conferencia de prensa o en un comunicado), media (refiere a hechos institucionales, aquí lo característico es que se trata de repertorios de acción normados desde las agencias estatales, como una presentación judicial o una reunión entre las partes en conflicto) y alta (refiere a hechos directos, esto es, hechos que implican la movilización de los cuerpos por fuera de los canales institucionales de negociación, como una manifestación, un corte de calle o una huelga). - Desobediencia: el objeto de esta variable es medir los grados de desobediencia (indisciplina) de los sujetos que emprenden la acción de rebelión. Permite establecer la proporción de acciones que se dan dentro del sistema institucional legal vigente y las que se dan por fuera de aquel sistema. En conjunto nos devuelve un mapa del respeto y transgresión de la legalidad burguesa. También se elabora a partir de la variable descriptiva "Forma de rebelión" y asume los valores: legal e ilegal.

- Energía que producen los cuerpos en lucha.

Nos parece importante detenernos y hacer una breve digresión en este punto, puesto que cuando en un artículo de ciencias sociales aparece el concepto de energía se dispara más de una alarma. Esto se debe en parte a la marginalidad del término en la narrativa que domina el campo académico. Si encima el término pretende ser 
utilizado como una variable con valores numéricos que permita medir la conflictividad social, el desafío se multiplica. Según sostiene Marín esta virtual ausencia de la noción de energía en el campo de las ciencias sociales se explica por su origen disciplinar, situación que implica un cierto retraso en su incorporación, como ha ocurrido con las nociones de masa, fuerza, etc. (Marín, 1995).

En primer lugar, tenemos que aclarar cómo entendemos el concepto en el contexto de este escrito. Siguiendo a Marín, sostenemos que la energía es aquello que busca ser expropiado de los cuerpos y monopolizado, proceso que hace a la dimensión poder, pues la energía de los cuerpos es equivalente a su poder potencial. Este ejercicio del poder expropiatorio y monopolizador persigue consumir "productivamente" la energía expropiada (Marín, 2009). En el marco de la relación capital/trabajo, los cuerpos proletarios, en tanto fuerza de trabajo asalariada, solo cuentan como energía en el proceso productivo social. Sin embargo, la imagen que tenemos sobre la energía es secularmente maniquea, pues aún hoy carecemos de un desarrollo conceptual del término a la altura de las necesidades de las ciencias sociales. Un sendero para seguir explorando lo abrió Marx en su análisis de la producción capitalista, en cuyo marco se producen a un tiempo las condiciones materiales de existencia biológica y, más importante aún, las relaciones sociales de existencia social, que hace a la dimensión valor/poder, como causa y consecuencia de la lucha de clases. Es en el proceso de producción y destrucción de lazos sociales donde el capital consume la energía material de los cuerpos, cuya resultante es la producción de valor/poder. Sin embargo, como toda

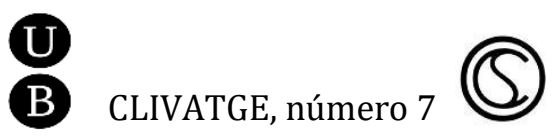


relación de poder, provoca resistencias y luchas (Marín, 1995).

Haciendo una analogía con el análisis de Marx (2004) en El Capital, podemos sostener que toda acción (trabajo humano) es gasto productivo ${ }^{9}$ del cerebro, músculo, nervio, mano, etc. La magnitud de ese gasto de energía se mide en la energía coagulada en la resultante de la acción (mercancía en un caso, evento de rebelión en otro). Es decir que la resultante representa trabajo humano puro y simple, gasto de energía corporal en general, coagulado en un evento de rebelión. Por esta razón consideramos que las acciones más complejas - por ejemplo, huelga general- deberían contar como acciones simples multiplicadas - por ejemplo, paros parciales en los lugares de trabajo-, de suerte que una pequeña cantidad de acciones complejas sea equivalente a una cantidad mayor de acciones simples. Por más que un evento de rebelión sea el producto de una acción más compleja, no representa más que determinada cantidad de acciones simples, o sea de gasto de energía. Así es que los distintos tipos de acciones son reducidos a acciones simples como unidad de medida del conflicto. Para simplificar, en lo sucesivo consideraremos directamente todo gasto de energía corporal como una acción simple, así la diferencia cualitativa se expresa en una diferencia cuantitativa, una diferencia en las magnitudes de la energía gastadas y coaguladas en el evento de rebelión. Son tratadas como acciones de igual carácter pero de diversa

\footnotetext{
${ }^{9}$ Productivo en un sentido distinto al expresado en El Capital; aquí refiere, por un lado, a la ruptura de relaciones sociales de disciplinamiento y obediencia al mando del capital y, por otro lado, a la creación de relaciones sociales de solidaridad y cooperación en contra de la dominación y la explotación capitalistas.
}

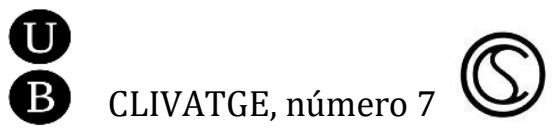


magnitud.

Retomemos ahora la explicación de la variable "Energía que producen los cuerpos en lucha". Con esta variable buscamos ponderar cualitativamente la magnitud de cada hecho en relación a los otros. ${ }^{10}$ Entendemos que este es un ejercicio de aproximación a la medición de la energía producida y usada, un indicador de la dimensión subjetiva de la lucha (grados de predisposición de los cuerpos a la lucha). Como venimos sosteniendo, los hechos que registramos en la base no son iguales, no implican lo mismo, por eso para su análisis es necesario medirlos en función de la magnitud de cada uno. La energía que producen los cuerpos en lucha es una variable que, al igual que otras, nos habla de la intensidad del hecho a partir de la forma y no en función sólo de la cantidad de cuerpos implicados. No es lo mismo el grado de cooperación y la fuerza material y moral que se requiere para difundir un comunicado de prensa que para llevar a cabo y sostener la toma de una empresa. Asimismo, la resistencia que opone el contrincante hace variar la magnitud de la energía, la cual se expresa en un cambio de forma de lucha. Para ponderar los hechos en función de la energía que implica cada uno aplicamos una escala geométrica: tomamos como "base" su cualidad de ser acciones de lucha contra el campo del régimen ${ }^{11}$ y luego establecemos multiplicaciones en función de las variables "nivel de implicancia corporal en la acción” y

\footnotetext{
${ }^{10}$ Replicamos parcialmente un ejercicio desarrollado en Beba Balvé (1990).

${ }^{11}$ A la inversa, en un análisis sobre las acciones del campo del régimen nuestra "base" es que son acciones contra el campo del pueblo.
}

B CLIVATGE, número 7

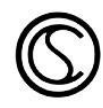


"desobediencia". Si el consenso social del régimen de dominación tiene valor 1,en la escala de producción de relaciones sociales reproductivas, cada hecho de rebelión representa una potencia multiplicadora diferente, a saber: hecho discursivo potencia por 2 el grado de cooperación para sí $\left(2^{1 *} 1\right)$; el hecho institucional lo potencia por $4\left(2^{2 *} 1\right)$; el hecho directo legal lo hace por $8\left(2^{3 *} 1\right)$; y el hecho directo ilegal lo hace por $16\left(2^{4 *} 1\right) .{ }^{12}$ Vale aclarar que a los fines de este ejercicio de aproximación consideramos a todos los sujetos de la rebelión como iguales, a pesar de que sabemos que no es así. Sin embargo, esta reducción no cambia el sentido de dicho ejercicio de aproximación.

A partir de estas variables analíticas, en las páginas siguientes se buscará restituir las heterogeneidades a fin de ponderar cualitativamente los datos que conforman la base de datos del SISMOS.

\section{De lo nacional a lo local y viceversa: Sobre los porqués de una base a ras del suelo}

Teniendo en cuenta que ya existe una base de datos sobre la conflictividad social general que registra eventos cubriendo todo el territorio nacional argentino a partir de información periódica

\footnotetext{
12 En ocasiones anteriores se nos ha interrogado acerca del porqué de la utilización de una escala geométrica y no otra. Destacamos en este sentido que lo numérico no es pertinente en sí (no se trata de porqué se elige la escala 2 - 4 - 8 - 16, en vez de 1 - 2 - 3-4, o cualquier otra); el recurso al número es el que nos permite, en el marco de un tratamiento estadístico de los datos, establecer el carácter relacional entre los diferentes formatos de acción, carácter que nos permite superar la igualación que hicimos de ellos para poder cargarlos en la base de datos (donde en expresión numérica serían todos equivalentes a 1).
}

B CLIVATGE, número 7

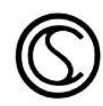


brindada por diarios capitalinos de alcance nacional y desde un marco teórico-conceptual afín al de SISMOS, cómo justificar, entonces, el solapamiento de los esfuerzos que significa la gestión de otra base de datos que solo es de alcance local. Este cuestionamiento se torna más relevante si, como efectivamente hacemos, se comparte la perspectiva que pondera como lo más importante de las bases sus relaciones, tendencias y valores relativos, mientras que los valores absolutos representan una jerarquía menor. El interrogante no es retórico, sino que pone en cuestión la necesidad científica de una base de datos de alcance local cuando ya existe una de alcance nacional que en su registro cubre todas las localidades del país, incluida la ciudad de Mar del Plata. Lo que sigue intenta ser una respuesta a este interrogante, a la vez que una presentación de la conflictividad social en su dimensión nacional a fin de referenciar lo registrado en la base local.

Entre enero de 2011 y junio de $2015^{13}$ (54 meses) el PIMSA registró en su base de datos 8.719 hechos de rebelión para el conjunto del territorio nacional. La media anual para el período 2011-2014 fue de 1.991 hechos. Como se ve en el Gráfico 1, el año con mayor frecuencia de hechos de rebelión fue el 2012 y el año 2011 fue el de menor frecuencia de hechos de rebelión. Por su parte, el bienio 20132014 muestra una tendencia a la baja en la frecuencia de hechos.

Si ahora contemplamos la relación entre la cantidad de hechos

\footnotetext{
${ }^{13}$ Los diferentes cortes temporales en las mediciones que se observan a lo largo del artículo responden a la disponibilidad de datos publicados por el PIMSA. Los datos fueron extraídos de http://www.pimsa.secyt.gov.ar/Inf.html [29-01-2019].
}

B CLIVATGE, número 7

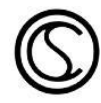


de rebelión distribuido porcentualmente entre 2011 y 2014 registrados por el PIMSA a escala nacional y por el SISMOS a escala local, notaremos una distribución relativa similar entre las dos series de datos para el mismo período de años. Las dos coinciden en el año de mayor y menor cantidad de hechos. También es coincidente la tendencia decreciente en la frecuencia del último bienio.

En este orden de las cosas y en relación al cuestionamiento inicial, los resultados de este ejercicio inclinan la balanza hacia la escasa relevancia de los aportes de una base local en la caracterización de la dinámica conflictual del período en cuestión, pues en términos relativos las trayectorias son análogas. Sin embargo, los datos referidos a MdP registrados en la base del PIMSA muestran una dinámica local divergente de la nacional. Esto ayuda a relativizar en alto grado la afirmación hecha en el párrafo precedente. Como se ve en el Gráfico 3, la silueta de la serie "PIMSAMdP" es divergente a la dibujada por las otras dos series. Esto se debe al mayor peso relativo de 2014 en la cantidad de hechos de rebelión de la serie. 
Gráficos 1, 2, 3: Frecuencia anual de HR total del país ${ }^{14}$ y Mar del Plata $^{15}$ según base de datos, 2011-2014
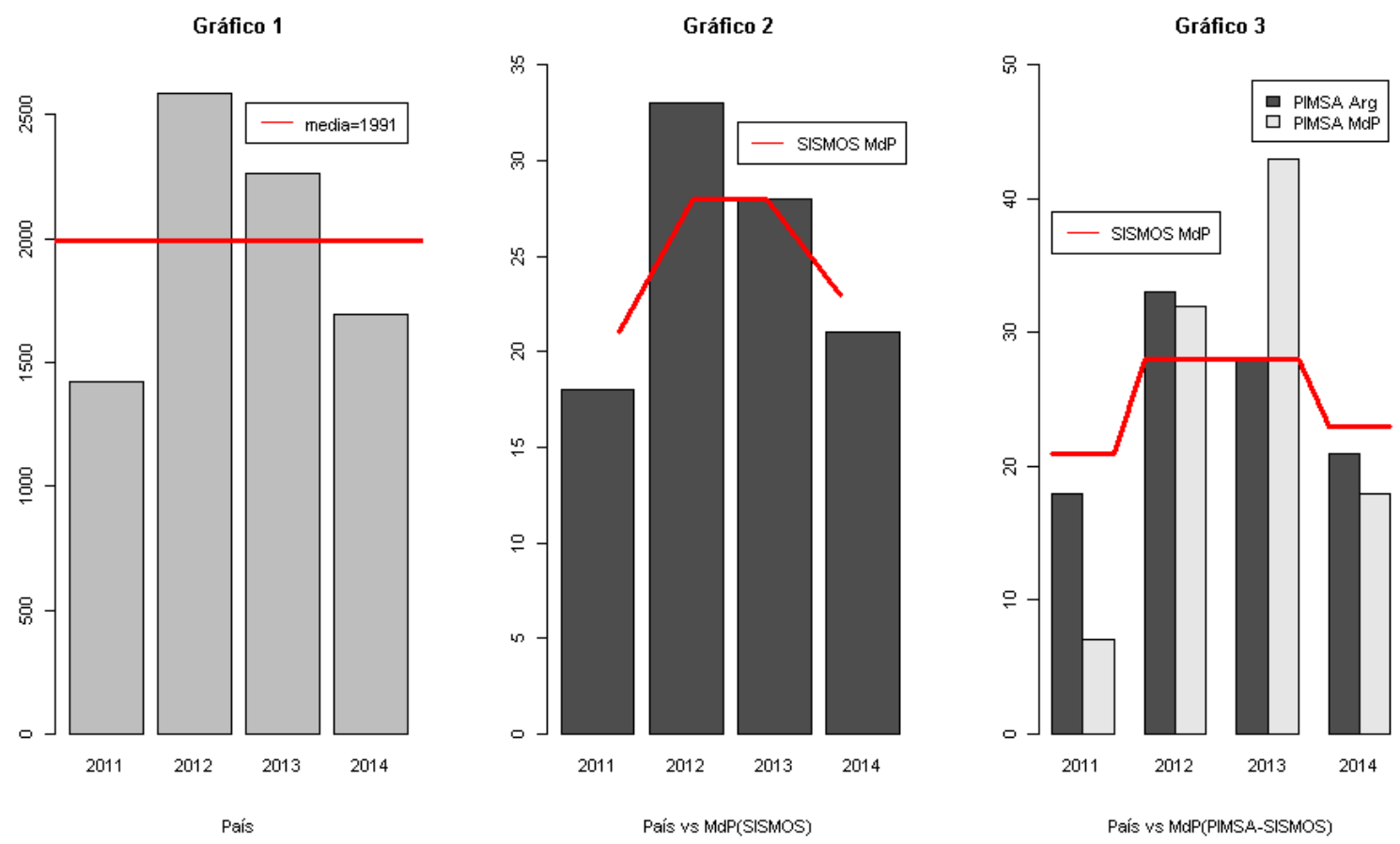

Fuente: PIMSA, SISMOS.

Como se ve, no sólo cambia el año pico sino que también lo hace la pendiente ascendente y descendente, en ambos casos más marcada, pues los años extremos tienen un valor porcentual más bajo que las otras dos series de datos. El dato más llamativo es el peso relativo del año inicial de la serie, con un $7,1 \%$ sobre el total de hechos del período,

\footnotetext{
${ }^{14}$ En los gráficos el total país se referencia como Arg.

${ }^{15}$ En los gráficos Mar del Plata se referencia como MdP.
}

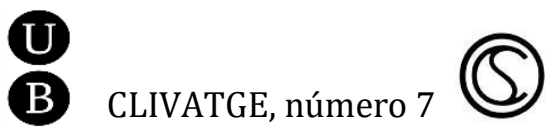


más de diez puntos por debajo que en las otras dos series.

Asimismo, a lo largo de estos cuatro años se nota, para todo el país, una tendencia a una mayor concentración de hechos protagonizados por asalariadxs en el primer semestre. Lo mismo ocurre si observamos la frecuencia de huelgas. A nivel nacional las huelgas se reparten semestralmente en la siguiente proporción: 61\% 1 semestre, 39\% 2 semestre (PIMSA); a nivel local su proporción es la siguiente: $60 \% 1$ semestre, $40 \% 2$ semestre (SISMOS). En parte esto se explica por el peso de las negociaciones paritarias del salario en el primer tramo del año. También incide el tiempo social del calendario cronológico anual, para el periodo bajo estudio notamos cierta rutinización anual de la lógica conflictual.

\section{Cuadro 1}

\section{Distribución de frecuencia de HR, total del país y MdP según} semestre, 2011-2014

\begin{tabular}{|l|l|l|l|l|l|l|}
\cline { 2 - 7 } \multicolumn{2}{|l}{} & \multicolumn{4}{|l}{ PIMSA } & \multicolumn{4}{l|}{ SISMOS } \\
\cline { 2 - 7 } \multicolumn{1}{|l|}{ Nacional } & \multicolumn{4}{l|}{ Mar del Plata } \\
\cline { 2 - 7 } & Asalariadxs & Otrxs & Asalariadxs & Otrxs & Asalariadxs & Otrxs \\
\hline 1 Semestre & 56 & 46 & 61 & 58 & 56 & 58 \\
\hline 2 Semestre & 44 & 54 & 39 & 42 & 44 & 42 \\
\hline Total & 100 & 100 & 100 & 100 & 100 & 100 \\
\hline
\end{tabular}

Fuente: PIMSA, SISMOS. 
Aunque con distinta intensidad y proporción, la distribución de frecuencia de hechos protagonizados por asalariadxs expresada en el Cuadro 1 muestra la incidencia mayor del primer semestre para el período 2011-2014 tomado en su conjunto. Esta situación, sumada a la similitud en la frecuencia semestral de huelgas, nuevamente pone en entredicho la significancia de una base de alcance local como la de SISMOS, pues más allá de algunos matices las tendencias generales parecen coincidir. Sin embargo, si cambiamos el ángulo de nuestro análisis y observamos la participación porcentual de cada tipo de sujeto sobre el total semestral, las tendencias divergen.

Si del Cuadro 2 leemos sólo la distribución de frecuencia de la base PIMSA para el total Nacional y Mar del Plata, las tendencias coinciden, con una mayor incidencia de Otrxs, tanto a nivel nacional como en Mar del Plata. Pero si incorporamos la distribución de frecuencia de la base SISMOS las tendencias cambian, se muestran diametralmente opuestas, con una incidencia mayor de "Asalariadxs". Esta constatación refuerza aún más la pertinencia de bases a ras del suelo. ${ }^{16}$

\footnotetext{
${ }^{16}$ Para el concepto a ras del suelo ver Nieto (2018).
}

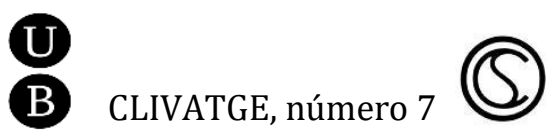




\section{Cuadro 2}

\section{Distribución de frecuencia de HR, total del país y MdP según}

sujeto, 2011-2014

\begin{tabular}{|c|c|c|c|c|c|c|}
\hline & \multicolumn{4}{|l|}{ PIMSA } & \multicolumn{2}{|l|}{ SISMOS } \\
\hline & \multicolumn{2}{|l|}{ Nacional } & \multicolumn{4}{|c|}{ Mar del Plata } \\
\hline & $\begin{array}{l}1 \\
\text { Semestre }\end{array}$ & $\begin{array}{l}2 \\
\text { Semestre }\end{array}$ & $\begin{array}{l}1 \\
\text { Semestre }\end{array}$ & $\begin{array}{l}2 \\
\text { Semestre }\end{array}$ & $\begin{array}{l}1 \\
\text { Semestre }\end{array}$ & $\begin{array}{l}2 \\
\text { Semestre }\end{array}$ \\
\hline Asalariadxs & 40 & 32 & 30 & 27 & 57 & 59 \\
\hline Otrxs & 60 & 68 & 70 & 73 & 43 & 41 \\
\hline Total & 100 & 100 & 100 & 100 & 100 & 100 \\
\hline
\end{tabular}

Fuente: PIMSA, SISMOS.

Antes de pasar al siguiente apartado nos interesa insistir en la importancia de emprender estudios locales y regionales amén de disponer del recorte nacional. A lo ya indicado en términos relativos debemos incorporar los datos en términos absolutos. Baste en este sentido apuntar que mientras la base del PIMSA para el periodo 20112015 registró un total de 144 hechos de rebelión en la ciudad de Mar del Plata, desde la base del SISMOS para el mismo período registramos un total de 2.645 acciones, las cuales se reducen a 1.085 hechos, los cuales a su vez disminuyen a 682 si filtramos hechos comunicacionales, ya que éstos no son registrados en la base de PIMSA. Algo más significativo aún, mientras que para el primer 
semestre de 2011 SISMOS registró 59 hechos (sin contar Comunicados) PIMSA no registró ningún hecho de rebelión. Si ahora desagregamos del total aquellos hechos emprendidos por asalariadxs encontramos que en el registro del PIMSA hay tres semestres sin ninguna acción para el período 2011-2015, mientras que la base de SISMOS tiene un piso de registro semestral de 25 hechos emprendidos por asalariadxs, como se puede observar en el Gráfico 4:

Distribución de frecuencia de HR por semestre en MdP según base y sujeto, 2011-2015

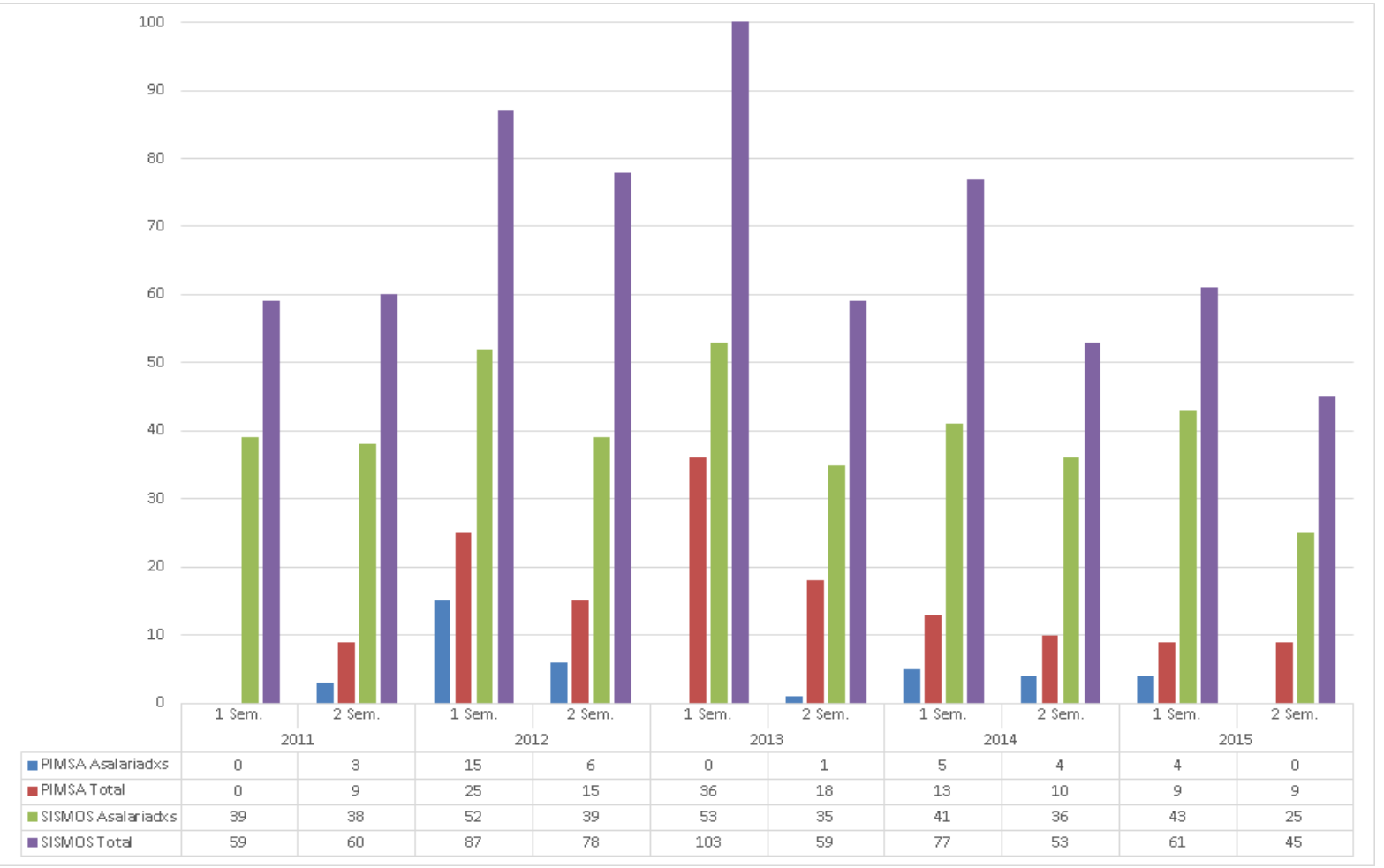

Fuente: PIMSA, SISMOS.

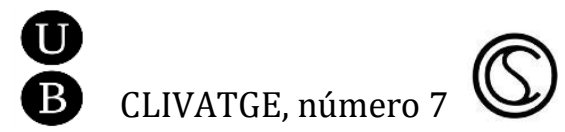


Por supuesto que estas observaciones no las realizamos con el propósito de ponderar qué es más útil, si un recorte local o uno nacional. Por el contrario, ambos son necesarios y complementarios para avanzar en el conocimiento acerca de las formas en que lo social - su orden y su desorden- deviene. Mientras que solo un recorte local nos habilita observar el conflicto en profundidad en la ciudad de Mar del Plata, el registro nacional nos permite reponer nuestros problemas de estudio en otra dimensión de análisis y, de este modo, esquivar sesgos producto de una distorsión 'localista'.

\section{Contabilizando la conflictividad social en el pago chico}

Antes de presentar el análisis del conflicto social a nivel local, es pertinente hacer una breve caracterización de la situación económica, laboral y política de Mar del Plata en el periodo bajo estudio. Con este objetivo a continuación presentamos una serie de indicadores básicos como la tasa de desempleo, el trabajo no registrado y la inflación.

Siguiendo los análisis presentados en los Informes Sociolaborales del Grupo de Estudios del Trabajo de la Universidad Nacional de Mar del Plata - elaborados conforme los datos publicados por la Encuesta Permanente de Hogares (EPH) del Instituto Nacional de Estadística y Censo (INDEC) de Argentina- en el periodo analizado la tasa de desempleo en el aglomerado Mar del Plata-Batán osciló entre el 7,8\% y el 12,1\%. El guarismo más bajo se registró durante el primer trimestre de 2011, mientras que el más alto durante

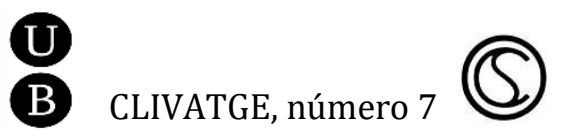


el tercer trimestre de 2016, prácticamente en los extremos del período (GrET, 2016, 2017). La tendencia de este indicador se manifiesta en ascenso. ${ }^{17}$ El guarismo más alto del periodo $(12,1 \%$ en el tercer trimestre de 2016) colocó a la ciudad de Mar del Plata en el primer puesto del "ranking" nacional de desempleo (GrET, 2016), si bien hay que aclarar que la ciudad se encuentra de manera estable dentro de las diez localidades con mayor desempleo a nivel país.

En el caso de Mar del Plata hay que tener en cuenta que junto a altas tasas de desempleo aparecen también tasas altas de empleo (entre 39,6\% y 44,7\% en el período) y de nivel de actividad (entre el $43.8 \%$ y el 49\%): "lo que sucede en Mar del Plata es que, si bien la cantidad de empleos es relativamente alta en comparación con otras ciudades, resulta insuficiente para incorporar a la cantidad de personas que buscan empleo" (GrET, 2014a: 21-22). Otra particularidad de la ciudad en relación a la tasa de desocupación es el sesgo de estacionalidad (por la actividad turística), producto del cual los valores más altos se presentan cuando se acerca la temporada de verano, mientras que los más bajos lo hacen en el resto del año (GrET, 2015: 22).

Por su parte, el trabajo no registrado — problemática central del mercado de trabajo argentino- en Mar del Plata-Batán, luego de los altos niveles que presentó en años anteriores en relación al total

\footnotetext{
${ }^{17}$ El período inmediatamente anterior fue de descenso si lo consideramos desde el pico de desocupación del segundo trimestre del 2009 que alcanzó el 14,4\% y llegó a 8,3\% en el segundo trimestre de 2010 (GrET, 2011).
}

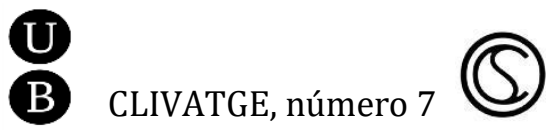


país (particularmente durante el 2009), con diferencias de alrededor del 10\%, muestra en el periodo 2011-2016 un proceso de estabilización aunque en niveles elevados "lo cual estaría indicando que esta problemática se encuentra ante un piso estructural" (GrET, 2014b: 23-24). ${ }^{18}$

Como último indicador de la situación económica, tengamos presente que la inflación ${ }^{19}$ en el período considerado fue en aumento (con la excepción de un descenso no menor en el año 2015), siendo la media de todo el periodo de $30,5 \%{ }^{20}$

Finalmente, en cuanto a la situación política institucional, hay que tener en cuenta que en el periodo de análisis aquí considerado hubo cambios en el gobierno de la administración estatal. El 2011, primer año de análisis, fue el último año del primer mandato del intendente de Mar del Plata Gustavo Pulti. Del 2012 a 2015 se cumplió su segundo mandato. Si bien procedente de un partido político local (Acción Marplatense), mantuvo fuertes vínculos con el gobierno provincial y el nacional de ese periodo (Frente para la Victoria,

\footnotetext{
${ }^{18}$ El porcentaje de trabajo no registrado oscila en el periodo entre $27,7 \%$ (segundo trimestre de 2012 ) y $36,5 \%$ (primer trimestre de 2012 ), siendo la media de $33,2 \%$, muy similar a la nacional (33,8\%) (GrET, 2015).

${ }^{19}$ En la Argentina, desde el fin del "Plan de Convertibilidad" en el año 2002 que mantenía el "1 a 1" entre el peso argentino y el dólar estadounidense, el tándem "devaluacióninflación es un problema constante de la economía que repercute fuertemente en la caída real de los salarios de Ixs trabajadorxs.

20 Según datos de IPC Congreso (extraído de http://www.ambito.com/diario/economia/banco-dedatos/bd_eco inflaci\%C3\%B3n_congreso.asp, última consulta 24/10/2018).
} 
dirigido en lo que refiere a su programa político por el Partido Justicialista, de signo peronista). Bonnet (2015) define este periodo de gobierno como una restauración del orden burgués que había sido astillado por la insurrección de 2001.

En diciembre de 2015 se produjo un cambio de gobierno en todos los niveles de la administración estatal (nacional, provincial y local). El frente político electo (Frente Cambiemos, integrado por los partidos políticos PRO [Propuesta Republicana] y UCR [Unión Cívica Radical]) presenta un programa de gobierno con fuertes pretensiones de restauración neoliberal. Desde su primer año de mandato (2016) avanzó sobre el recorte de derechos (en salud, educación), despidos en las agencias estatales y destrucción de puestos de trabajo en el sector privado (de la mano de la apertura de las importaciones), recorte y paralización de programas sociales, reposicionamiento del capital financiero, entre otras medidas.

Introduciéndonos de lleno en los datos registrados en nuestra base, realizamos ahora una primera lectura descriptiva de la conflictividad en Mar del Plata. Durante el período 2011-2016 se registraron un total de 3.718 acciones conflictivas que equivalen a 1.436 hechos de rebelión. La distribución de los 1.436 hechos por año es de $12 \%$ en el $2011,20 \%$ en el $2012,13 \%$ en el $2013,12 \%$ en el $2014,14 \%$ en el 2015, y finalmente $29 \%$ en el 2016. Si observamos los años correspondientes a los meses finales del primer mandato del intendente Gustavo Pulti en la ciudad (2011), más los cuatro años de su segundo mandato (2012-2015), se observa que el año con mayor frecuencia de hechos fue el 2012, mientras que el resto se mantuvo

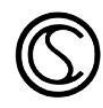


relativamente estable, pero con un leve aumento del $2 \%$ hacia el final del periodo (año 2015). Respecto al año 2016 vale resaltar que presenta un alto porcentaje de hechos en relación con los demás. En efecto, presenta 9 puntos porcentuales más (29\%) que el valor más alto de la serie hasta 2015 (20\% en 2012). Con lo cual el 2016 se presenta como el año con mayor caudal de hechos de rebelión de la serie. Esto implica que 2016 redundó en un porcentaje que duplicó el caudal de HR del resto de los años, con la excepción de 2012. Aunque siguió la tendencia general de una baja en la frecuencia de HR en el segundo semestre del año, la reducción no fue significativa. En relación a esta tendencia, la única excepción fue el año 2011, año en el cual el segundo semestre mostró una frecuencia mayor de HR que el primer semestre.

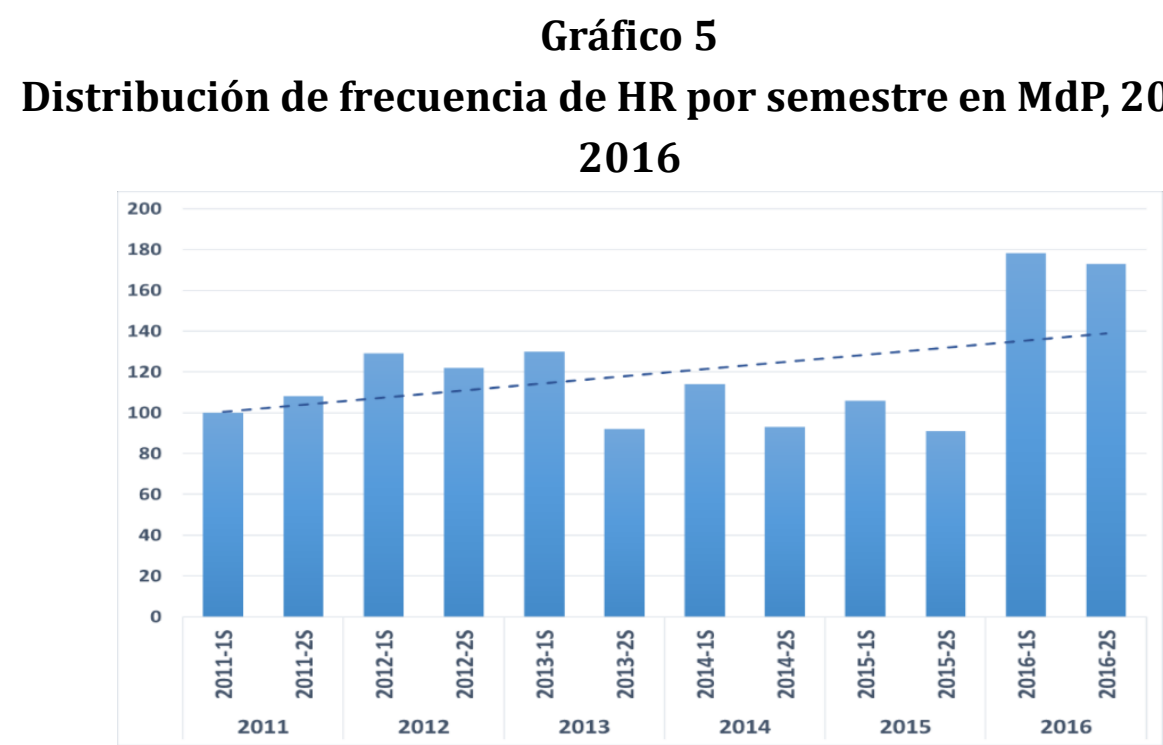

Fuente: SISMOS.
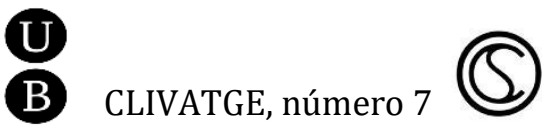
Si ponemos en relación los datos locales recién presentados con los del apartado anterior - nacionales-, distribuidos a lo largo de los nueve semestres que componen el período enero de 2011 y junio de 2015 , se observan siluetas mayormente contrapuestas, salvo para tres intersemestrales: del $2^{\circ}$ semestre de 2011 al $1^{\circ}$ semestre de 2012, del $1^{\circ}$ al $2^{\circ}$ semestre de 2014 y de este último al $1^{\circ}$ de 2015 . Conjeturamos que esto se debe al peso relativo que en cada serie tienen los hechos emprendidos por asalariadxs en el universo de eventos registrados. Al ser mayor el peso de lxs asalariadxs en el total de hechos registrados por SISMOS, y al estar estos vinculados en gran medida a las negociaciones paritarias anuales, los primeros semestres de la serie tienen, salvo para el 2011, una frecuencia mayor que los segundos semestres. A la inversa ocurre con la base del PIMSA, salvo para el año 2011 y el 2014. Gráfico 6: Distribución de frecuencia de HR por semestre para el total del país y Mar del Plata, 2011-2015

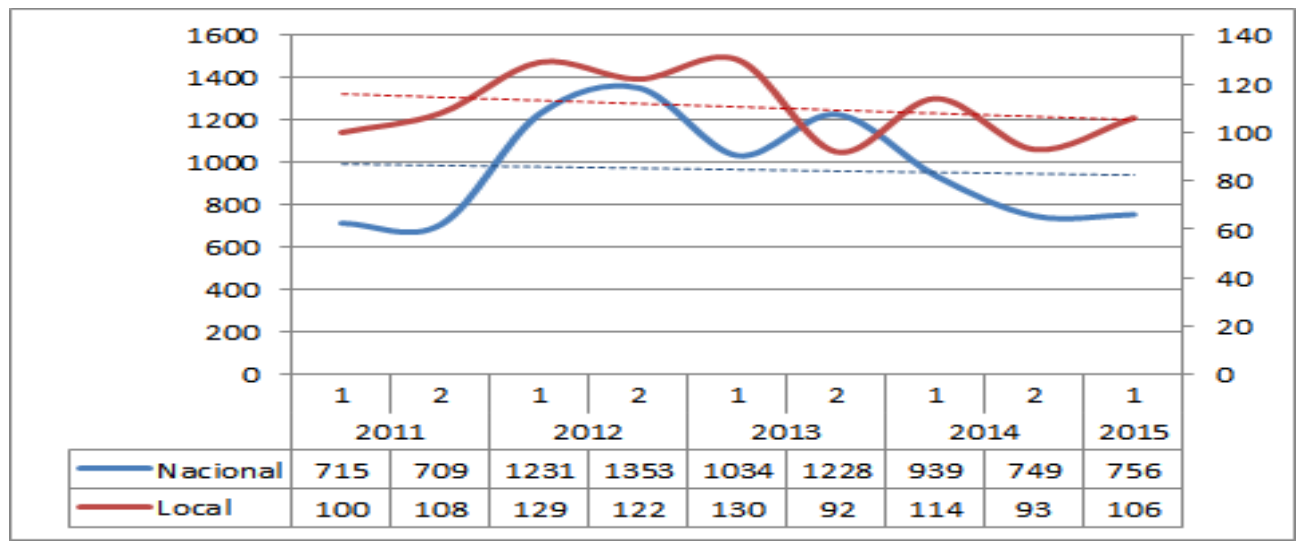

Fuente: PIMSA, SISMOS. 
Ahora bien, si nos detenemos ya no en los comportamientos en términos absolutos sino en las tendencias (ver líneas de punto en el Gráfico 6) se observa que ambos registros presentan una tendencia decreciente aunque, con pendientes distintas, más pronunciada en el caso de Mar del Plata. La tendencia en esta última se invierte cuando incorporamos a la serie el año 2016 (Gráfico 5). Aquí conjeturamos que el cambio en el signo político de la gestión del gobierno del estado en la ciudad, la provincia y el país modificaron las condiciones de la dinámica conflictual, tanto en su cantidad como en su contenido. Esto no debe ser leído como un cambio en el marco de oportunidades políticas, concepto propio de la teoría de la acción colectiva, sino como el emergente de un proceso de lucha política entre dos fuerzas sociales mayoritarias, ambas de carácter eminentemente burgués pero de composición policlasista (Cotarelo, 2016). La forma principal en este proceso de lucha política fue (y sigue siendo) la electoral. 0 sea, es un proceso que se da dentro de la legalidad burguesa por integrarse en mejores condiciones en el orden institucional vigente. La diferencia es que se pasa de un momento de tregua inestable a un período de confrontación abierta cuyas consecuencias son inciertas. 21 Conjeturamos que desde el cambio de gobierno el momento es de confrontación abierta porque la fuerza social del régimen se hizo con el gobierno del estado y está intentando realizar su victoria electoral

${ }^{21}$ Como parte de esta conjetura, sostenemos que la tregua se construyó a partir de 2003/2004 y empezó a resquebrajarse hacia 2008/2009.

B CLIVATGE, número 7

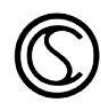


en el plano social, económico y cultural.

Pasando ahora al tipo de sujeto que emprende los HR, se observa que del total de hechos 58\% fueron emprendidos por sujetos que expresan PRK/T, mientras que $42 \%$ fueron impulsados por sujetos que expresan PORS. En relación con los primeros, las actividades del ámbito estatal como la educación, la salud, la administración pública y la justicia concentraron el activismo sindical fue más dinámico. En el ámbito privado lxs trabajadorxs de la pesca, del transporte urbano, de los balnearios, de la alimentación, del comercio y de la energía fueron lxs más activos. En relación con los segundos, los sectores más activos fueron lxs estudiantes, junto a lxs vecinxs, las comunidades educativas y las organizaciones políticopartidarias. Observemos ahora el comportamiento de ambos sujetos con relación a la forma que adoptan los conflictos.

En relación a los sujetos que expresan PRK/T, como se puede ver en el Cuadro 3, cerca del $80 \%$ de los hechos se distribuyen entre tres formas de rebelión, a saber, comunicados, huelga y manifestación callejera. Si marginamos momentáneamente del análisis los hechos exclusivamente discursivos, la huelga emerge como la principal forma de lucha de lxs asalariadxs, seguida de lejos por la manifestación callejera. Por su parte, los cortes aparecen muy por debajo de las huelgas con un $2 \%$, mientras que las ocupaciones quedan agrupadas en la categoría "Otras" por su escaso peso porcentual. 


\section{Cuadro 3}

Distribución de frecuencia de HR impulsados por PRK/T según forma de rebelión, Mar del Plata 2011-2016

\begin{tabular}{lll} 
Forma de rebelión & $\mathrm{N}^{\circ}$ & $\%$ \\
\hline Comunicados & 330 & $40 \%$ \\
Huelga & 230 & $28 \%$ \\
Manifestación callejera & 91 & $11 \%$ \\
Reunión entre partes litigantes & 49 & $6 \%$ \\
Asamblea, Reunión & 33 & $4 \%$ \\
Estado de alerta & 22 & $3 \%$ \\
Corte de calle, Corte de ruta & 19 & $2 \%$ \\
Ocupación & 13 & $2 \%$ \\
Otras & 35 & $4 \%$ \\
\hline Total general & 822 & $100 \%$ \\
\hline
\end{tabular}

Fuente: SISMOS.

Es interesante también la relación entre los HR según el sector en donde emergen. Se observa que, mientras el $50 \%$ de los hechos que producen los sujetos de la relación $\mathrm{K} / \mathrm{T}$ se emprenden desde el sector privado, el $37 \%$ lo hace desde el sector público, un $12 \%$ son hechos 
emprendidos mancomunadamente y un 1\% reviste la categoría de sin datos. Esta supremacía del sector privado se revierte si discriminamos entre asalariadxs y empresarixs, estos últimos emprendieron el 12\% de los hechos. De esta forma la relación entre asalariadxs del ámbito privado y del estatal queda equiparada con un 36\% y un $36 \%$.

Como se puede observar en el Cuadro 4, al igual que para los hechos emprendidos por PRK/T, cerca del $80 \%$ de los hechos impulsado por PORS se distribuyen entre tres formas de rebelión, a saber: comunicados, manifestación callejera y cortes. Si marginamos momentáneamente del análisis los hechos exclusivamente discursivos, la manifestación callejera emerge como la principal forma de lucha de estas otras personificaciones, seguida de lejos por el corte de calles o rutas. Es significativo notar que emergen con valores iguales o mayores a $2 \%$ categorías que estaban subsumidas en "Otras" en el Cuadro 3, nos referimos a ataques, enfrentamientos y acciones judiciales.

\section{Cuadro 4}

\section{Distribución de frecuencia de HR impulsados por PORS según forma de rebelión, Mar del Plata 2011-2016}

\begin{tabular}{lll} 
Forma de rebelión & $\mathrm{N}^{\circ}$ & $\%$ \\
\hline Comunicados & 217 & $37 \%$ \\
Manifestación callejera & 185 & $31 \%$ \\
Corte de calle, Corte de ruta & 55 & $\mathbf{9 \%}$
\end{tabular}

B CLIVATGE, número 7

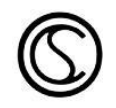




\begin{tabular}{lcc|} 
Ataque & 36 & $6 \%$ \\
Reunión entre partes litigantes & 28 & $5 \%$ \\
Ocupación & 17 & $3 \%$ \\
Asamblea, Reunión & 15 & $3 \%$ \\
Acción judicial & 14 & $2 \%$ \\
Enfrentamientos & 10 & $2 \%$ \\
Otras & 14 & $2 \%$ \\
\hline Total general & 591 & $100 \%$ \\
\hline \multicolumn{2}{c}{ Fuente: SISMOS. } \\
\hline
\end{tabular}

Es interesante notar que en ambos cuadros la frecuencia más alta corresponde a la categoría comunicados, con una proporcionalidad muy similar. Esto nos permite conjeturar que la confrontación discursiva, sin ser mayoritaria, es la base de las otras formas de rebelión con independencia del sujeto que la emprende. Decimos esto porque, en las otras formas de rebelión el elemento discursivo también está presente aunque no sea el objetivo directo de la acción. 0 sea, no hay acción de rebelión que no implique de algún modo un repertorio discursivo, como marco legitimador.

Por otro lado, es interesante observar aquí un poco más de cerca el tipo de sujeto que emprende los hechos de rebelión, superando la definición de un colectivo Otros, homogeneizado en su construcción en tanto negativo de las PRK/T. Particularmente nos 
interesa observar quienes dentro de este grupo expresan la identidad de vecinxs, puesto que una de nuestras hipótesis de trabajo sostiene que por fuera de la matriz sindical predominante encontramos una impronta muy fuerte de acciones callejeras de entidades barriales y vecinales de corte fomentista, lo que responde a una larga tradición de activismo vecinal y barrial en sociedades de fomento y vecinales en el sudeste bonaerense, la cual es constantemente reactualizada. En efecto, del total de los hechos de rebelión llevados a cabo por personificaciones que se expresan por fuera de la relación $\mathrm{K} / \mathrm{T}$, el $40 \%$ corresponde a la categoría Vecinxs.. Cuadro 5:

Frecuencia de hechos impulsados por vecinxs según forma de rebelión, Mar del Plata 2011-2016.

\begin{tabular}{lll} 
Forma de rebelión & $\mathrm{N}^{\circ}$ & $\%$ \\
\hline Comunicados & 94 & $41 \%$ \\
Manifestación callejera & 49 & $21 \%$ \\
Reunión entre partes litigantes & 26 & $11 \%$ \\
Corte de calle, Corte de ruta & 21 & $9 \%$ \\
Ataque & 16 & $7 \%$ \\
Ocupación & 6 & $3 \%$ \\
Asamblea, Reunión & 5 & $2 \%$ \\
Acción judicial & 5 & $2 \%$ \\
Otras & 8 & $4 \%$ \\
\hline Total general & 230 & $100 \%$ \\
\hline
\end{tabular}

Fuente: SISMOS.

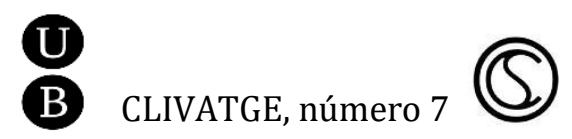


Con este último cuadro cerramos parcialmente el recorrido por las frecuencias de hechos según formas de rebelión y sujeto en clave descriptiva. Sin embargo, nos interesa concluir este apartado con un breve análisis de las acciones emprendidas por vecinxs y las emprendidas por PRK/T, y una conjetura a ser desarrollada en los próximos párrafos. Si comparamos los datos de este último cuadro con los datos del Cuadro 3, en lo que hace a hechos cuyas acciones principales fueron directas e ilegales —ataque, ocupación, corte-, notaremos que, de conjunto, vecinxs y sujetos que PRK/T llevaron adelante 77 hechos de este tipo. De este conjunto 34 (44\%) fueron emprendidos por PRK/T y 43 (56\%) por vecinxs. Si ahora calculamos el peso relativo que este tipo de hechos tiene sobre el conjunto de hechos llevados a cabo por cada una de estas personificaciones el resultado es el siguiente: $4 \%$ en el total de hechos protagonizados por PRK/T y 19\% en el total de hechos protagonizados por vecinxs. Esto nos permite adelantar la siguiente conjetura: lxs vecinxs, en tanto PORS, muestran una predisposición mayor que lxs asalariadxs a llevar a cabo acciones directas de carácter ilegal. A qué se debe esta diferencia lo intentaremos abordar en el siguiente apartado.

\section{Midiendo la conflictividad social a ras del suelo: algunos ejercicios de ponderación}

Como reza su título, en este apartado nos abocaremos a presentar algunos ejercicios de ponderación para el período 20112016 en la ciudad de Mar del Plata. En primer lugar, presentaremos la

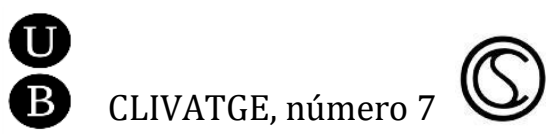


frecuencia anual de hechos del período según grado de desobediencia e implicancia corporal de los sujetos de la rebelión. Si bien este primer ejercicio no es en sí mismo una ponderación, nos permite reagrupar y simplificar la presentación de la serie de datos analizados.

Un primer emergente de este reagrupamiento es el lugar que ocupan los HR que implican acciones directas legales (Alta-Legal) en relación a aquellos HR que solo implican expresiones discursivas (Baja) para el total de HR del período. Mientras que en la distribución según forma de rebelión la mayor frecuencia la detentaba la categoría Comunicados (aquí agrupada en la categoría Baja), en la distribución según grado de desobediencia e implicancia corporal, los hechos discursivos pasan a detentar un peso similar a las acciones directas agrupadas en el valor Alta-Legal. 


\section{Cuadro 6}

\section{Distribución de frecuencia de HR según grado de desobediencia e implicancia corporal, Mar del Plata 2011-2016, $(n=1436)$}

\begin{tabular}{|c|c|c|c|c|c|c|c|}
\hline $\begin{array}{l}\text { Grado de desobediencia e } \\
\text { implicancia corporal }\end{array}$ & 2011 & 2012 & 2013 & 2014 & 2015 & 2016 & Total \\
\hline $\begin{array}{l}\text { Alta-Ilegal (relativo a los otros } \\
\text { valores) }\end{array}$ & $9 \%$ & $9 \%$ & $14 \%$ & $13 \%$ & $7 \%$ & $14 \%$ & $11 \%$ \\
\hline $\begin{array}{l}\text { Alta-Ilegal (relativo a los otros } \\
\text { años) }\end{array}$ & $12 \%$ & $14 \%$ & $18 \%$ & $17 \%$ & $9 \%$ & $30 \%$ & $100 \%$ \\
\hline $\begin{array}{l}\text { Alta-Legal (relativo a los otros } \\
\text { valores) }\end{array}$ & $39 \%$ & $43 \%$ & $48 \%$ & $39 \%$ & $39 \%$ & $33 \%$ & $40 \%$ \\
\hline $\begin{array}{l}\text { Alta-Legal (relativo a los otros } \\
\text { años) }\end{array}$ & $14 \%$ & $19 \%$ & $19 \%$ & $14 \%$ & $14 \%$ & $20 \%$ & $100 \%$ \\
\hline $\begin{array}{l}\text { Media (relativo a los otros } \\
\text { valores) }\end{array}$ & $8 \%$ & $9 \%$ & $7 \%$ & $10 \%$ & $7 \%$ & $8 \%$ & $8 \%$ \\
\hline Media (relativo a los otros años) & $14 \%$ & $20 \%$ & $14 \%$ & $17 \%$ & $11 \%$ & $24 \%$ & $100 \%$ \\
\hline Baja (relativo a los otros valores) & $44 \%$ & $39 \%$ & $31 \%$ & $38 \%$ & $47 \%$ & $45 \%$ & $41 \%$ \\
\hline Baja (relativo a los otros años) & $15 \%$ & $17 \%$ & $12 \%$ & $13 \%$ & $16 \%$ & $27 \%$ & $100 \%$ \\
\hline Total & $100 \%$ & $100 \%$ & $100 \%$ & $100 \%$ & $100 \%$ & $100 \%$ & $100 \%$ \\
\hline
\end{tabular}

Fuente: SISMOS.

\footnotetext{
${ }^{22}$ Esta variable expresa de modo conjunto a las variables: grado de implicancia corporal y grado de desobediencia. La primera asume tres valores (baja - donde se agrupa a las acciones comunicacionales -, media - donde se agrupa a las acciones institucionales - , y alta - donde se agrupa a las acciones directas-). La segunda asume dos valores: legal e ilegal (este último valor incluye sólo al conjunto de acciones que asumen el valor alta en el grado de implicancia corporal y que se dan por fuera del sistema legal. Con todo ello, la variable aquí presentada puede asumir cuatro valores: alta ilegal, alta legal, media o baja.
} 
Si observamos la distribución de los HR según grado de desobediencia e implicancia corporal en cada uno de los años bajo estudio, notamos que hay variaciones interanuales de importancia. Por ejemplo, en 2013 los valores relativos a las series de las categorías Alta-Legal y Baja muestran los valores extremos del período bajo estudio, con el valor más alto (48\%) y el más bajo (31\%) respectivamente. Por su parte, el año 2015 muestra el valor más alto de la serie en lo que hace al peso proporcional de la categoría Baja, con un $47 \%$ sobre el total de hechos de ese año.

Si observamos el comportamiento de cada uno de los valores que expresan los grados de desobediencia-implicancia de los cuerpos en la rebelión a lo largo del período, podemos ver que los HR que agrupan acciones directas legales presentan una fuerte caída en 2014, razón por la cual la serie presenta una tendencia decreciente que comienza a revertirse en 2016. Por su parte, los HR que agrupan acciones discursivas (desobediencia-implicancia corporal Baja) muestran un comportamiento más oscilante, aunque también con una tendencia decreciente que comienza a revertirse en 2016. En 2013 esta serie tiene su valor más bajo (12\%).

Los HR que agrupan acciones directas ilegales (Alta-Ilegal) muestran un comportamiento oscilante, aunque también con una tendencia decreciente que comienza a revertirse en 2016. En 2015 esta serie tiene su valor más bajo (9\%). Finalmente, los HR que agrupan acciones institucionales judiciales y/o de negociación (Media) muestran un comportamiento oscilante, aunque con una

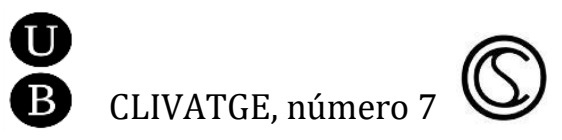


tendencia creciente que no parece revertirse en el primer semestre de 2016. En 2015 esta serie tiene su valor más bajo (11\%).

Veamos ahora qué sucede cuando introducimos el ejercicio de ponderación de la energía desplegada en los HR.

Como había ocurrido con el reagrupamiento según grado de desobediencia-implicancia corporal, los resultados de la ponderación arrojan una nueva modificación en el orden de las categorías. Los dos datos más relevantes son el ascenso de los HR que implican acciones directas ilegales al segundo lugar y el descenso de los HR que implican sólo expresiones discursivas al tercer lugar, mientras que los extremos se mantienen en el mismo rango. Por otra parte, en relación a la categoría Alta-Ilegal la energía desplegada se incrementa a partir de 2013.

\section{Cuadro 7}

Distribución de frecuencia de HR ponderados según grado de desobediencia-implicancia corporal, Mar del Plata 2011-2016, en porcentajes (columnas).

\begin{tabular}{|c|c|c|c|c|c|c|c|}
\hline & 2011 & 2012 & 2013 & 2014 & 2015 & 2016 & Total \\
\hline Alta-Ilegal & $26 \%$ & $24 \%$ & $31 \%$ & $33 \%$ & $22 \%$ & $34 \%$ & $29 \%$ \\
\hline Alta-Legal & $52 \%$ & $57 \%$ & $56 \%$ & $49 \%$ & $56 \%$ & $49 \%$ & $53 \%$ \\
\hline Media & $7 \%$ & $6 \%$ & $4 \%$ & $6 \%$ & $5 \%$ & $5 \%$ & $6 \%$ \\
\hline Baja & $15 \%$ & $13 \%$ & $9 \%$ & $12 \%$ & $17 \%$ & $12 \%$ & $13 \%$ \\
\hline Total & $100 \%$ & $100 \%$ & $100 \%$ & $100 \%$ & $100 \%$ & $100 \%$ & $100 \%$ \\
\hline
\end{tabular}

B Clivatge, número 7 (S) 
Pasemos a ver ahora qué sucede cuando discriminamos entre hechos ponderados emprendidos por SPRK/T y hechos emprendidos por SPORS.

Antes de analizar el cuadro recordemos que los hechos emprendidos por SPRK/T representan el 58\% de los hechos totales, mientras que los SPORS impulsaron el restante $42 \%$. Como vemos en el Cuadro 8, salvo para una de las cuatro categorías, la energía desplegada por los SPRK/T en los procesos de rebelión tienen un piso del $61 \%$ en relación al total de energía desplegada, mientras que el piso para los SPORS es del 35\%. Sin embargo, cuando leemos el valor total de cada una de las dos columnas la relación es de 54/46. Esto se explica por el peso relativo de esa cuarta categoría, la que refiere a los hechos que implican acciones directas ilegales, pues aquí las proporciones se invierten a favor de las personificaciones de otras relaciones sociales. Es esta relación 26/74 la que explica la paridad en la reducción porcentual de 16 puntos a 8 cuando medimos la energía desplegada (54/46) en vez de contabilizar hechos (58/42). 0 sea que con un caudal menor de hechos los SPORS despliegan un caudal de energía cercano al desplegado por los SPRK/T. Esto significa que la media de energía desplegada por HR es mayor para los SPORS y menor para los SPRK/T. 


\section{Cuadro 8}

Distribución de frecuencia de hechos ponderados según grado de desobediencia e implicancia corporal discriminado por tipo de sujeto, Mar del Plata 2011-2016

\begin{tabular}{|ll|ll|} 
& & K/T & Otras \\
\hline \multirow{2}{*}{ Alta-Legal } & (s/sujeto) & $65 \%$ & $35 \%$ \\
& (s/tipo de H) & $65 \%$ & $40 \%$ \\
Alta-Ilegal & (s/sujeto) & $\mathbf{2 6 \%}$ & $\mathbf{7 4 \%}$ \\
& (s/tipo de H) & $\mathbf{1 4 \%}$ & $\mathbf{4 5 \%}$ \\
Baja & (s/sujeto) & $64 \%$ & $36 \%$ \\
& (s/tipo de H) & $15 \%$ & $10 \%$ \\
Media & (s/sujeto) & $61 \%$ & $39 \%$ \\
& (s/tipo de H) & $6 \%$ & $5 \%$ \\
& \multicolumn{2}{c}{ Fuente: SISMOS. } \\
\end{tabular}

Esto se conecta con las reflexiones realizadas en el final del apartado anterior, donde destacamos el mayor peso de las acciones directas ilegales, como las ocupaciones, los cortes y los ataques, en el conjunto de HR impulsados por "vecinxs". Finalmente, la comparativa de la energía desplegada discriminada entre los dos tipos de sujetos implicados tanto en lo que hace a la proporción de ese tipo de hechos dentro del conjunto de hechos impulsados por cada uno de los dos 
sujetos como a la proporción dentro del mismo tipo de hechos entre ambos sujetos, nos permite sostener que la alta radicalidad de las acciones pertenecientes a los conflictos no laborales es uno de sus rasgos característicos. Como sostiene Piva, "la usual apelación al corte de ruta, calle o de vías de trenes en la mayoría de los casos muestra la generalización de la apelación a la acción directa radical. Pero también resultan significativas medidas más duras como la ocupación e incluso el ataque de edificios públicos" (2011: 13).

Estos resultados preliminares disparan al menos un par de interrogantes: ¿Qué lleva a vecinxs, fomentistas, familiares y otras personificaciones no identificadas de forma directa con la relación $\mathrm{K} / \mathrm{T}$ a tener que desplegar mayor energía que aquellas identidades que personifican la relación $\mathrm{K} / \mathrm{T}$, y viceversa? ¿Este despliegue es producto de su fortaleza o de su debilidad estructural? ¿Existe correspondencia entre radicalidad en la forma de rebelión y radicalidad en las metas perseguidas? ¿Es este comportamiento producto de una mayor o una menor "integración" social? Finalmente, ¿este despliegue está vinculado a una escasa o nula institucionalización/normalización de sus formas de lucha, contrario a lo que sucede, por ejemplo, con la huelga? 


\section{Cuadro 9}

Frecuencia de hechos ponderados según energía emprendidos por SPRK/T, Mar del Plata 2011-2016, en porcentajes

\begin{tabular}{l|cccccc|c}
$\begin{array}{l}\text { Grado de } \\
\text { desobedienciae } \\
\text { implicancia }\end{array}$ & $\mathbf{2 0 1 1}$ & $\mathbf{2 0 1 2}$ & $\mathbf{2 0 1 3}$ & $\mathbf{2 0 1 4}$ & $\mathbf{2 0 1 5}$ & $\mathbf{2 0 1 6}$ & Total \\
\hline $\begin{array}{l}\text { Alta-Ilegal (relativo } \\
\text { a los otros valores) }\end{array}$ & $17 \%$ & $9 \%$ & $17 \%$ & $13 \%$ & $10 \%$ & $19 \%$ & $14 \%$ \\
$\begin{array}{l}\text { Alta-Ilegal (relativo } \\
\text { a los otros años) }\end{array}$ & $24 \%$ & $11 \%$ & $22 \%$ & $16 \%$ & $11 \%$ & $16 \%$ & $100 \%$ \\
\hline $\begin{array}{l}\text { Alta-Legal (relativo } \\
\text { a los otros valores) }\end{array}$ & $62 \%$ & $70 \%$ & $68 \%$ & $67 \%$ & $63 \%$ & $58 \%$ & $65 \%$ \\
$\begin{array}{l}\text { Alta-Legal (relativo } \\
\text { a los otros años) }\end{array}$ & $16 \%$ & $20 \%$ & $20 \%$ & $18 \%$ & $15 \%$ & $11 \%$ & $100 \%$ \\
\hline $\begin{array}{l}\text { Media (relativo a } \\
\text { los otros valores) }\end{array}$ & $4 \%$ & $8 \%$ & $3 \%$ & $5 \%$ & $8 \%$ & $6 \%$ & $6 \%$ \\
$\begin{array}{l}\text { Media (relativo a } \\
\text { los otros años) }\end{array}$ & $20 \%$ & $23 \%$ & $9 \%$ & $17 \%$ & $18 \%$ & $13 \%$ & $100 \%$ \\
\hline $\begin{array}{l}\text { Baja (relativo a los } \\
\text { otros valores) }\end{array}$ & $17 \%$ & $13 \%$ & $12 \%$ & $15 \%$ & $19 \%$ & $18 \%$ & $15 \%$ \\
$\begin{array}{l}\text { Baja (relativo a los } \\
\text { otros años) }\end{array}$ & $21 \%$ & $15 \%$ & $15 \%$ & $17 \%$ & $19 \%$ & $13 \%$ & $100 \%$ \\
\hline $\begin{array}{l}\text { Total } \\
\text { 100\% }\end{array}$ & $\mathbf{1 0 0 \%}$ & $\mathbf{1 0 0 \%}$ & $\mathbf{1 0 0 \%}$ & $\mathbf{1 0 0 \%}$ & $\mathbf{1 0 0 \%}$ & $\mathbf{1 0 0 \%}$
\end{tabular}

Fuente: SISMOS. 
A modo de conjetura consideramos que el mayor despliegue de energía por parte de los SPORS responde a su debilidad estructural, su inestable "integración" social y la carencia de una forma de lucha normalizada. Esto nos lleva a considerar que su radicalidad se corresponde más con esta situación que con las metas perseguidas.

Pasemos a analizar ahora la distribución de frecuencias de la energía desplegada según año y tipo de sujeto.

Para el caso de SPRK/T (Cuadro 9), una de las primeras características que emergen del análisis del cuadro es la concentración de energía en los hechos que agrupan acciones directas legales (65\%) con una variación interanual no muy oscilante. Por otra parte, la energía desplegada en el marco de este tipo de HR presenta un descenso a partir del 2013. En lo que hace a la energía concentrada por los HR que agrupan acciones directas ilegales y discursivas, su participación gira en torno al 15\%, con oscilaciones interanuales de significancia. Finalmente, la energía concentrada por los HR que agrupan las acciones institucionales gira en torno a un $6 \%$ aunque con variaciones interanuales muy grandes.

Para el caso de SPORS (Cuadro 10), una de las primeras características que emerge del análisis del cuadro es la concentración de energía en torno a dos tipos de hechos que suman el 85\%, los que agrupan acciones directas ilegales (45\%) y los que agrupan acciones directas legales (40\%), con una variación interanual bastante oscilante. Por otra parte, el segundo trienio presenta un ascenso en la energía desplegada en el marco de los hechos que agrupan acciones directas ilegales. En lo que hace a la energía desplegada por los hechos

B CLIVATGE, número 7

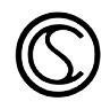


que agrupan acciones directas legales el segundo trienio presenta una caída en relación al primer trienio. El restante 14\% se distribuye entre las otras dos categorías: $10 \%$ para hechos discursivos y 5\% para hechos institucionales. Ambos presentan variaciones interanuales muy grandes, siendo un rasgo llamativo el valor porcentual de $1 \%$ para el año 2015 en referencia a la energía desplegada por los hechos institucionales.

Los últimos párrafos los dedicaremos a analizar los HR en función de otras dimensiones. Con tal objetivo realizaremos otro ejercicio de ponderación que no toma como variable base la forma de rebelión sino que se basa en la dimensión temporal y la cantidad de acciones por hecho. En base a las variables "duración temporal del hecho" y "cantidad de acciones por hecho de rebelión" desarrollamos una ponderación en función de cuatro categorías: Alta duración y Baja duración (que corresponden los valores de la variable "duración temporal del hecho") y Alto $\mathrm{n}^{\circ}$ de acciones y Bajo $\mathrm{n}^{\circ}$ de acciones (que corresponden a los valores de la variable "cantidad de acciones por hecho de rebelión"). La división entre alto y bajo la establecimos en función de la media para cada una de las dos variables. Así logramos esquematizar en cuatro cuadrantes el ejercicio de ponderación: A, B, C, D. Estos cuadrantes son producto de la combinación de las cuatro categorías antes mencionadas: A=Alta/Bajo; $\mathrm{B}=$ Alta/Alto; C=Baja/Bajo; D= Baja/Alto.

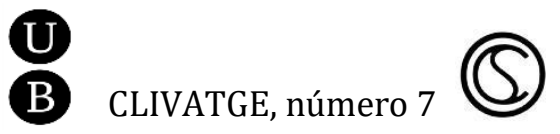




\section{Cuadro 10}

Frecuencia de hechos ponderados según energía emprendidos por SPORS, Mar del Plata 2011-2016, en porcentajes

\begin{tabular}{|c|c|c|c|c|c|c|c|}
\hline $\begin{array}{l}\text { Grado de } \\
\text { desobediencia } \\
\text { e implicancia }\end{array}$ & 2011 & 2012 & 2013 & 2014 & 2015 & 2016 & Total \\
\hline $\begin{array}{l}\text { Alta-Ilegal } \\
\text { (relativo a los } \\
\text { otros valores) }\end{array}$ & $38 \%$ & $37 \%$ & $47 \%$ & $59 \%$ & $40 \%$ & $49 \%$ & $45 \%$ \\
\hline $\begin{array}{l}\text { Alta-Ilegal } \\
\text { (relativo a los } \\
\text { otros años) }\end{array}$ & $11 \%$ & $17 \%$ & $21 \%$ & $21 \%$ & $11 \%$ & $19 \%$ & $100 \%$ \\
\hline $\begin{array}{l}\text { Alta-Legal } \\
\text { (relativo a los } \\
\text { otros valores) }\end{array}$ & $45 \%$ & $43 \%$ & $27 \%$ & $46 \%$ & $38 \%$ & $45 \%$ & $40 \%$ \\
\hline $\begin{array}{l}\text { Alta-Legal } \\
\text { (relativo a los } \\
\text { otros años) }\end{array}$ & $13 \%$ & $22,5 \%$ & $22,5 \%$ & $11 \%$ & $14 \%$ & $17 \%$ & $100 \%$ \\
\hline $\begin{array}{l}\text { Media (relativo } \\
\text { a los otros } \\
\text { valores) }\end{array}$ & $8 \%$ & $4 \%$ & $5 \%$ & $6 \%$ & $1 \%$ & $5 \%$ & $5 \%$ \\
\hline $\begin{array}{l}\text { Media (relativo } \\
\text { a los otros } \\
\text { años) }\end{array}$ & $21 \%$ & $18 \%$ & $21 \%$ & $21 \%$ & $1 \%$ & $18 \%$ & $100 \%$ \\
\hline
\end{tabular}




\begin{tabular}{l|ccccccc}
\hline $\begin{array}{l}\text { Baja (relativo a } \\
\text { los otros } \\
\text { valores) }\end{array}$ & $13 \%$ & $13 \%$ & $5 \%$ & $8 \%$ & $13 \%$ & $9 \%$ & $10 \%$ \\
$\begin{array}{l}\text { Baja (relativo a } \\
\text { los otros años) }\end{array}$ & $17 \%$ & $27 \%$ & $11 \%$ & $13 \%$ & $16 \%$ & $16 \%$ & $100 \%$ \\
\hline $\begin{array}{l}\text { Total } \\
\mathbf{1 0 0 \%}\end{array}$ & $\mathbf{1 0 0 \%}$ & $\mathbf{1 0 0 \%}$ & $\mathbf{1 0 0 \%}$ & $\mathbf{1 0 0 \%}$ & $\mathbf{1 0 0 \%}$ & $\mathbf{1 0 0 \%}$ \\
\hline
\end{tabular}

Fuente: SISMOS.

De esta forma, en base a un diagrama cartesiano, distribuimos el espectro de intensidad de los HR en cuatro cuadrantes, donde la flecha roja indica el mayor o menor grado de intensidad de los hechos según su ubicación en los cuadrantes.

\section{Gráfico 7}

Diagrama para la distribución de los HR según días y acciones afectadas por $\mathrm{HR}$

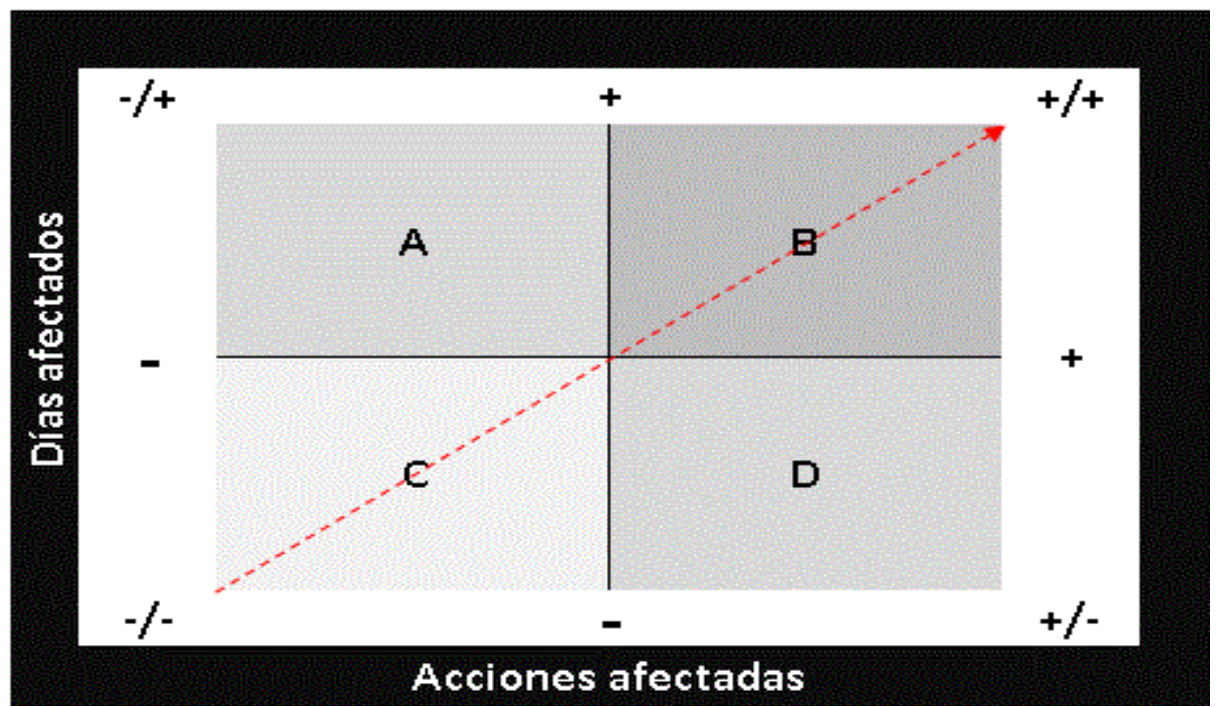

B CLIVATGE, número 7

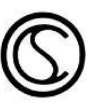


Para la realización de este ejercicio de ponderación sólo tomamos HR que agrupaban dos o más acciones (es decir, hechos de rebelión compuestos, en adelante, HRC), razón por la cual el valor mínimo del eje x (Acciones afectadas) es 2 y el valor máximo se obtiene de la cantidad de acciones que concentra el hecho de mayor agregación de acciones del espectro estudiado. Por su parte, el eje y (Días afectados) tiene como valor mínimo 1, y como valor máximo la cantidad de días que concentra el hecho de mayor duración. Asimismo, debido a la diferencia entre la serie de valores de los dos ejes, establecimos una escala logarítmica de base 10. El resultado, expresado en un gráfico de dispersión con escalas logarítmicas, fue el siguiente:

Lo primero que notamos es que el cuadrante $\mathrm{C}$ concentra la mayor proporción de HRC (60\%). 0 sea que la mayor parte de los HRC tienen una duración menor a 34 días y agrupan menos de 5 acciones por hecho compuesto. Sin embargo, el cuadrante B, que agrupa los HRC de mayor intensidad, detenta el segundo lugar con el 17\% de los HRC. Al tomar los HRC del cuadrante B (es decir, aquellos más intensos en función de su duración temporal y de la cantidad de acciones que agrupan) vemos cómo los últimos 6 semestres (2014-2016) presentan, con oscilaciones, una tendencia ascendente, distinta a la dibujada por la frecuencia de HR sin discriminar según temporalidad ni $\mathrm{N}^{\circ}$ de acciones agrupadas por HR. Finalmente, este ejercicio abona la conjetura del cambio de dinámica conflictual hacia el final del período bajo estudio, momento en el cual los conflictos se tornan más

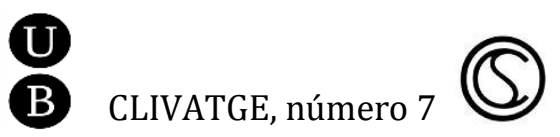


intensos. Esto se debe a un cambio en la correlación de fuerzas en el estado del poder entre las clases sociales.

\section{Gráficos 8, 9: Dispersión y Distribución de frecuencia según duración y cantidad de acciones}
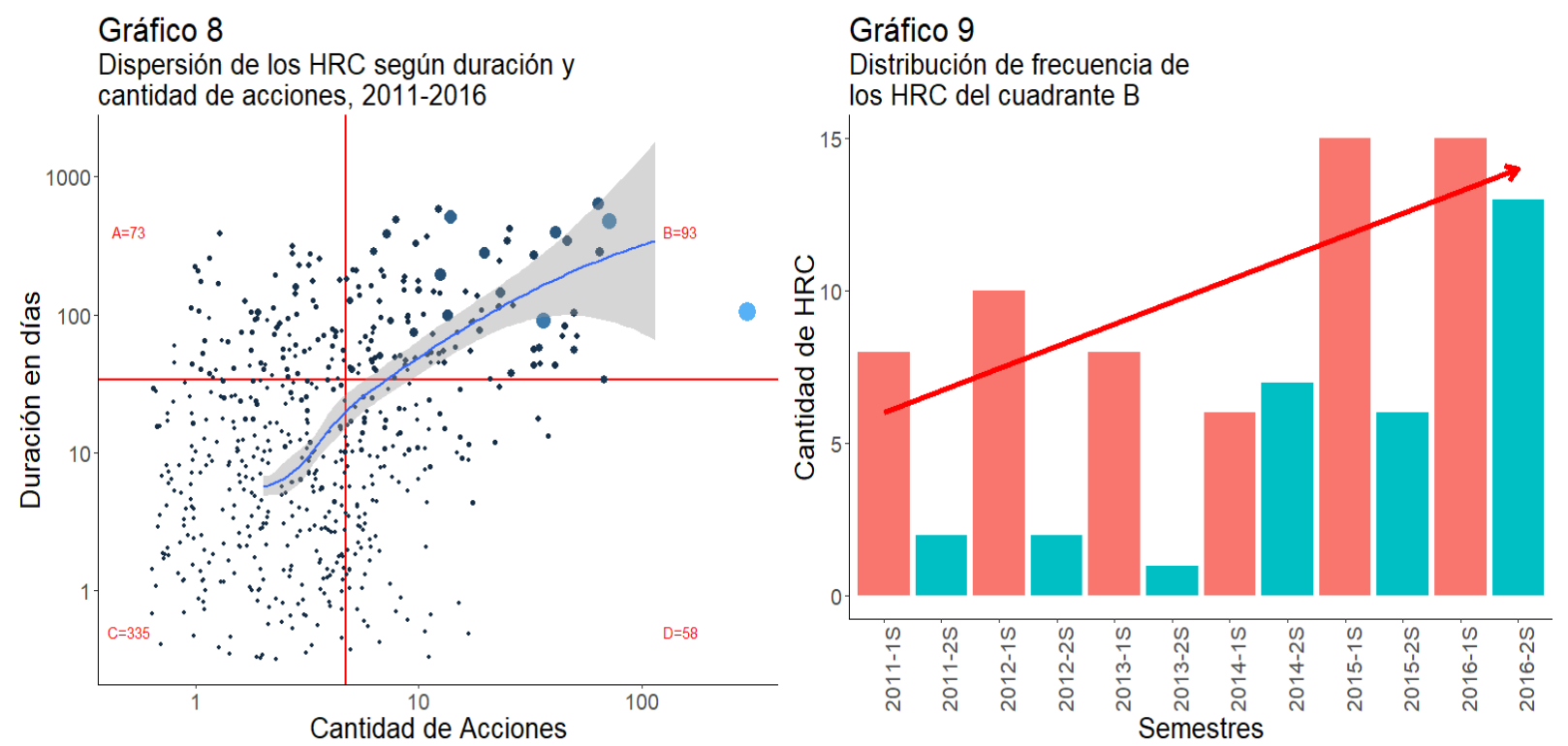

Fuente: SISMOS.

\section{Conclusiones}

Después de una larga exposición y análisis de gráficos y cuadros pudimos llegar a los siguientes resultados preliminares y parciales:

1. En la ciudad-puerto de Mar del Plata, durante el período bajo estudio, existió una tendencia a la concentración de hechos en el
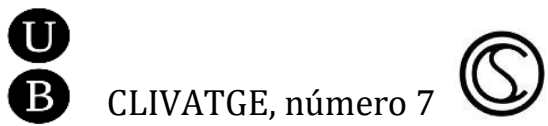
primer semestre del año, que difiere de lo que muestra la base de datos del PIMSA para el conjunto del país. Esto responde, por un lado, a cierta correspondencia entre los tiempos sociales, institucionales sobre todo, y el calendario. Por otro lado, responde a la periodicidad de las negociaciones paritarias que, salvo excepciones, se realizan durante la primera mitad del año. Para poner a prueba esta tesis, en futuros avances contrastaremos este período con la década de 1990, donde las negociaciones paritarias centralizadas y anuales no estaban vigentes.

2. Vinculado a esto último, podemos afirmar que la mayoría de los hechos fueron impulsados y protagonizados por asalariadxs. Cuando acotamos la distribución semestral a las personificaciones de la relación $\mathrm{K} / \mathrm{T}$, la tendencia a la concentración de hechos en el primer semestre se profundiza. Nuevamente, esta tesis será testeada contrastando este período con un período de auge del movimiento de desocupados (década de 1990).

3. El ejercicio de ponderación de la energía desplegada en los hechos de rebelión nos permitió confirmar que la cantidad de hechos no es un buen indicador de intensidad de la rebelión. Asimismo, nos permitió constatar que los sujetos que emprenden menos acciones no necesariamente despliegan un caudal menor de energía, sino que en ocasiones ocurre lo contrario. Finalmente, gracias a este ejercicio pudimos ver que las personificaciones de otras relaciones sociales desarrollan menos cantidad de acciones pero de mayor despliegue de energía, debido esto al peso específico y relativo de las acciones directas ilegales. Si bien para este período el foco estuvo en "Vecinxs",

B CLIVATGE, número 7

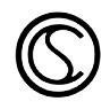


en próximos avances pondremos a prueba esta idea con el análisis del tipo de acciones emprendidas por desocupadxs y trabajadorxs "informales".

4. A partir de la dimensión temporal, gracias al ejercicio de ponderación que puso en relación las variables "días afectados" y "acciones afectadas", notamos que las tendencias iniciadas entre 2013 y 2014 se vieron profundizadas con el cambio de gobierno y su consecuente reconfiguración de las correlaciones de fuerzas. Este resultado preliminar logrará ser testeado cuando contemos con más datos sobre el periodo en curso (2016-2019).

En un próximo artículo tenemos pensado complementar nuestro análisis con la incorporación de la dimensión "tipo de demanda", ya que, a partir de algunos indicios, conjeturamos que desde 2014 a 2016 existe una tendencia creciente de las demandas de carácter defensivo (es decir, acciones de rebelión que se activan no por la conquista de nuevos derechos, sino para defender los vigentes). Asimismo, estamos avanzando en el registro de las acciones de rebelión en Necochea, ciudad cercana a Mar del Plata, lo que nos permitirá contrastar las dinámicas conflictivas en ambas ciudadespuerto del sudeste de la provincia de Buenos Aires. 


\section{Bibliografía}

Balvé, Beba et al., (1990): La función de la huelga en el proceso de formación de fuerza social. Cuaderno CICSO, Buenos Aires. Serie Estudios n 65.

Balvé, Beba y Beatriz Balvé (1989): El '69. Huelga política de masas. Rosariazo, cordobazo, rosariazo. Contrapunto, Buenos Aires.

Bonnet, Alberto (2015): La insurrección como restauración. El kirchnerismo, 2002-2015. Buenos Aires: Prometeo.

Chakrabarty, Dipesh (2008): "La historia subalterna como pensamiento político". En Mezzadra, Sandro (comp), Estudios postcoloniales. Ensayos fundamentales. Traficantes de sueños, Madrid, pp. 145-165

Cotarelo, María Celia (2016): Argentina (1993-2010). El proceso de formación de una fuerza social. Imago Mundi, Buenos Aires.

Edwards, P. K. y Hugh Scullion (1987): La organización social del conflicto laboral: control y resistencia en la fábrica. Ministerio de Trabajo y Seguridad Social, Madrid.

Franzosi, Roberto (2017): “La prensa como fuente de datos socio-históricos: Cuestiones sobre la Metodología de recolección de datos a partir de periódicos", Revista de estudios Marítimos y Sociales, no 11, Mar del Plata, pp. $255-286$

Ghigliani, Pablo (2009): “Acerca de los estudios cuantitativos sobre conflictos laborales en Argentina (1973-2009): reflexiones sobre sus premisas teórico-metodológicas", Conflicto Social, año 2, n 2, diciembre, Buenos Aires, pp. 76-97

Gramsci, Antonio (2010): Antología. Siglo XXI Editores, Buenos Aires.

GrET (2011): Informe Sociolaboral del Partido de General Pueyrredón, $\mathrm{n}^{\circ}$ 6, julio, Grupo de Estudios del Trabajo, Mar del Plata, disponible en http://nulan.mdp.edu.ar/1369/22/03/2017

GrET (2014a): Informe Sociolaboral del Partido de General Pueyrredón, ${ }^{\circ}{ }^{\circ} 11$, junio, Grupo de Estudios del Trabajo, Mar del Plata, disponible en 
http://nulan.mdp.edu.ar/1971/22/03/2017

GrET (2014b): Informe Sociolaboral del Partido de General Pueyrredón, ${ }^{\circ} 12$, diciembre, Grupo de Estudios del Trabajo, Mar del Plata, disponible en http://nulan.mdp.edu.ar/2094/22/03/2017.

GrET (2015): Informe Sociolaboral del Partido de General Pueyrredón, $\mathrm{n}^{\circ} 13$, octubre, Grupo de Estudios del Trabajo, Mar del Plata, disponible en http://nulan.mdp.edu.ar/2395/, 22/03/2017

GrET (2016): Informe Sociolaboral del Partido de General Pueyrredón, ${ }^{\circ} 15$, diciembre, Grupo de Estudios del Trabajo, Mar del Plata, disponible en http://nulan.mdp.edu.ar/2589/22/03/2017

GrET (2017): Informe Sociolaboral del Partido de General Pueyrredón, $\mathrm{n}^{\circ} 16$, marzo, Grupo de Estudios del Trabajo, Mar del Plata, disponible en http://nulan.mdp.edu.ar/2626/, 24/10/2018

Guixá, Federico, Laitano, Guillermina, Nieto, Agustín y Nicolás Rabino (2015): "La conflictividad social en Mar del Plata en la historia reciente. Un ejercicio de medición". Publicado en Actas de las II Jornadas de Jóvenes Investigadores del Centro de Estudios Históricos, Facultad de Humanidades, UNMdP, Mar del Plata.

Laitano, Guillermina, Nicolás Rabino y Agustín Nieto (2015): "Procesos de rebelión social: ejercicios de medición y ponderación”. Ponencia presentada en el Panel "Construcción de datos del conflicto laboral", Segundo Taller Conflicto Laboral: problemas teóricos y metodológicos, organizado por CEIL - CONICET, Capital Federal, viernes 13 de noviembre.

Maañón, Mariana y Flabián Nievas (2003): “El problema de la medición de los conflictos sociales". Ponencia presentada en el Congreso de la Asociación Latinoamericana de Sociología, Perú.

Marín, Juan Carlos (1995): Conversaciones sobre el poder. Instituto de Investigaciones Gino Germani, Buenos Aires. 
Marín, Juan Carlos (2009): Leyendo a Clausewitz. Cuaderno 8. Ediciones PICASO, Buenos Aires.

Marx, Karl (2004): El Capital. Siglo XXI, Buenos Aires.

Millán, Mariano (2007): "Una propuesta para la medición de los conflictos sociales: la unidad ponderada de conflicto social". Ponencia presentada en las VII Jornadas de Sociología de la UBA, Buenos Aires.

Nieto, Agustín (2018): Entre anarquistas y peronistas. Historias obreras a ras del suelo. Ediciones CEHTI, Ediciones Imago Mundi, Buenos Aires.

Sorribas, Patricia (2010): "Acciones colectivas en torno a lo laboral. Algunos planteos metodológicos", Boletín Onteaiken, año 5, n 9, Córdoba, pp. 6475. En línea: http://onteaiken.com.ar/ver/boletin9/2-1.pdf, $(13 / 06 / 14)$.

Wright, Erik Olin (2010): "Comprender la clase: hacia un planteamiento analítico integrado", New Left Review, n 60, Madrid, pp. 98-112. 